\title{
Reflections on Paul Erdős on His Birth Centenary
}

Krishnaswami Alladi and Steven Krantz, Coordinating Editors

\begin{abstract}
This is Part I of a two-part feature on Paul Erdős following his centennial. There are eleven articles by leading experts who have reflected on the remarkable life, contributions, and influence of this towering figure of twentieth century mathematics. Here in Part I we have contributions from Krishnaswami Alladi and Steven Krantz, László Lovász and Vera T. Sós, Ronald Graham and Joel Spencer, Jean-Pierre Kahane, and Mel Nathanson.

Part II will contain articles by Noga Alon, Dan Goldston, András Sárközy, József Szabados, Gérald Tenenbaum, and Stephan Garcia and Amy Shoemaker.
\end{abstract}

\section{Krishnaswami Alladi and Steven Krantz}

\section{One of the Most Influential Mathematicians of Our Time}

The 100th birth anniversary of the great Hungarian mathematician Paul Erdős was celebrated in Budapest in July 2013 with an international conference that attracted about 750 participants. Erdôs was one of the most influential mathematicians of the twentieth century for a variety of reasons. He made fundamental and pioneering contributions in several fields of mathematics, such as number theory, combinatorics, graph theory, analysis, geometry, and set theory. He was perhaps the most prolific mathematician in history after Euler, with more than 1,500 papers, but what was most interesting about this was that more than half of these were joint papers and many of his collaborators were very young. Thus through the fundamental ideas in his papers and through these

Krishnaswami Alladi is professor of mathematics at the University of Florida. His email address is a11 adik@uf1 . edu.

Steven Krantz is professor of mathematics at Washington University in St. Louis. His email address is sk@math .wust 1 . edu.

DOI: http://dx.doi.org/10.1090/noti1211

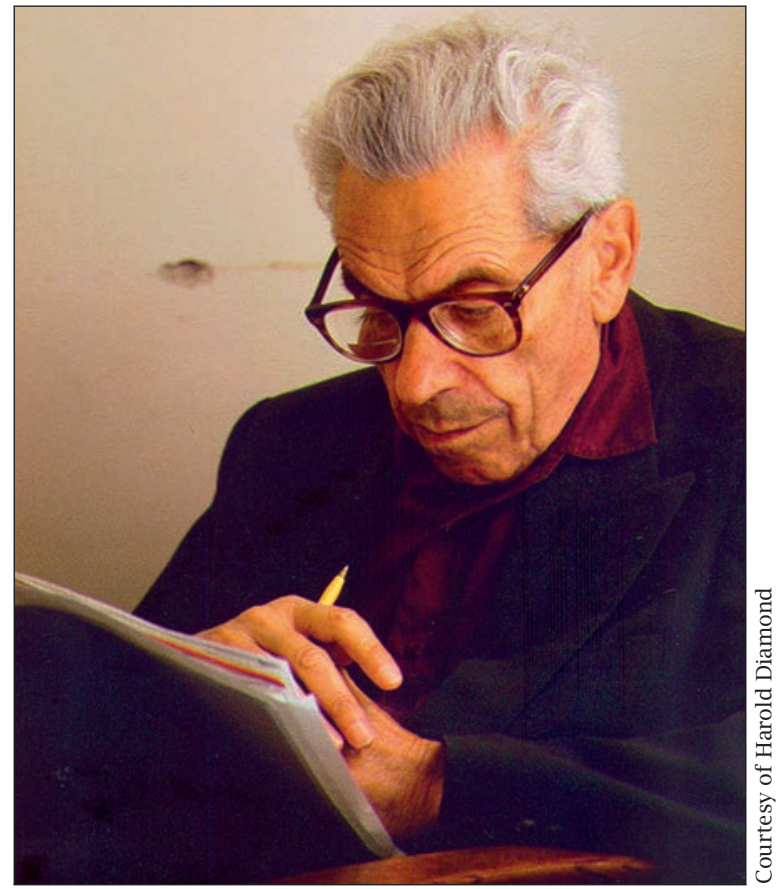

Paul Erdős working in his office at the Hungarian Academy of Sciences, Budapest.

collaborations, he influenced several generations of mathematicians and molded the careers of many. He was the greatest problem proposer in history, 


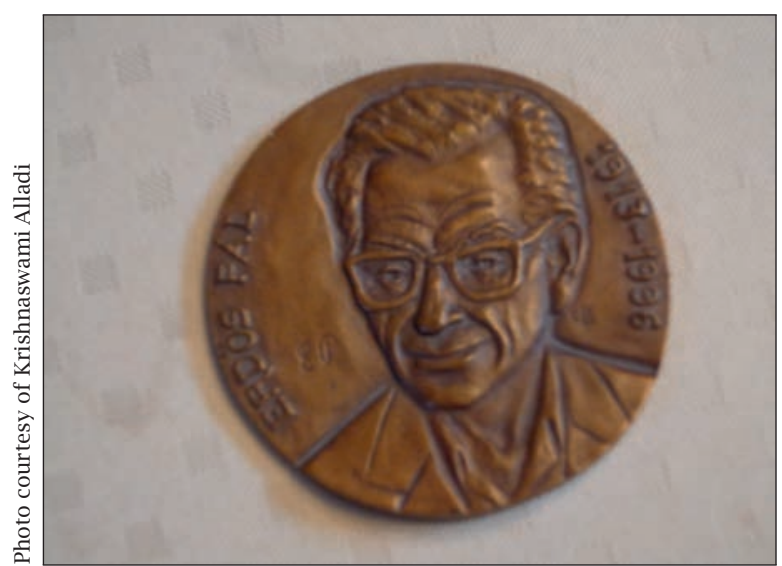

An emblem of Erdős made for the Erdős Memorial Conference in Budapest, Hungary, July

1999.

and for many of his problems he offered prize money depending on their difficulty. These problems have shaped the development of several areas, and most have remained unsolved. The lectures at the Erdôs Centennial Conference demonstrated that the influence of Paul Erdôs on the growth of mathematics remains strong. Here we have gathered eleven articles by leading mathematicians on various aspects of Erdős's work and his influence on current research. In our article we make some personal reflections, touch upon some of Erdős's work in number theory, and describe ways in which his legacy has been honored.

Like all great geniuses, Erdôs had his idiosyncrasies. But even in a mathematical world used to the peculiarities of its luminaries, Erdós was a most unusual phenomenon. There are hundreds of Erdős stories that are fondly recalled at various mathematical gatherings, and this feature has a good sampling of such recollections.

Like many of the greatest mathematicians in history, Erdós made his entry into the world of research very early in his life and in a grand manner. His very first paper [4] was a proof of Bertrand's postulate, which states that for any $n \geq 1$, there is always a prime number in the interval $[n, 2 n]$. The Russian mathematician Chebychev was the first to prove this, but Erdôs's proof utilizing the study of the prime factors of the middle binomial coefficient is so elegant and clever that it is this proof that is given in all textbooks on number theory. News of Erdós's proof spread like wildfire and was accompanied by a rhyme:"Chebychev said it and I say it again, there is always a prime between $n$ and $2 n$." During many of his lectures, and in particular in an article entitled "Ramanujan and I" [7], published on the occasion of the Ramanujan Centennial, Erdős has pointed out that there are similarities between his proof of Bertrand's postulate and Ramanujan's, but he was not aware of Ramanujan's proof when he wrote his first paper in 1932.

Erdős has often jokingly said that God has a book of the most beautiful proofs of the most important theorems. His desire was to glance through this book of God (after death)! It should be pointed out that Erdôs was an atheist and referred to God as the Supreme Fascist. But in the case of the proofs, he admitted that God possessed this wonderful Book. Martin Aigner and Gunter Ziegler have brought out a publication [1] entitled Proofs from The Book containing the most beautiful proofs of important theorems in various branches of mathematics. There are several proofs of Erdős in this book, since his proofs are extremely clever, elegant, and elementary.

A famous problem of Erdős which is still unsolved and for which he has offered US $\$ 3,000$ is the following: If $\left\{a_{n}\right\}$ is a sequence of increasing positive integers such that $\sum \frac{1}{a_{n}}$ is divergent, then prove that the sequence $\left\{a_{n}\right\}$ contains arbitrarily long arithmetic progressions. An important special case of this problem when the $\left\{a_{n}\right\}$ is the sequence of primes has been settled, and this is the celebrated Green-Tao theorem [11]. In 1936 Erdôs and Turán conjectured that if $\left\{a_{n}\right\}$ has upper positive density, then $\left\{a_{n}\right\}$ contains arbitrarily long arithmetic progressions. Erdôs offered US $\$ 1,000$ for the resolution of this conjecture, which was proved by Szemerédi [20].

Prime numbers were among Erdős's favorite topics of investigation. The prime number theorem, which states that the number of primes up to $x$ is asymptotic to $x / \log x$, implies that the average gap between the $n$th prime $p_{n}$ and the next one is asymptotic to $\log n$. Two questions immediately arise: (i) Can the ratio $r_{n}=\left(p_{n+1}-p_{n}\right) / \log n$ be arbitrarily large? (ii) How small can this ratio be infinitely often? The unsolved prime twins conjecture is the extreme solution to (ii). The recent sensational result of Zhang [23] on bounded gaps between primes shows that the ratio is $O(1 / \log n)$ infinitely often (there is more about this in Goldston's article in Part II of this feature. See also Note Added in Proof to this article). The famous US $\$ 10,000$ problem of Erdós concerns (i). Westzynthius [22] in 1931 showed that the ratio $r_{n}$ can be arbitrarily large. Subsequently, the Scottish mathematician Rankin [17] was able to show more precisely that there exists a constant c such that $r_{n}>\frac{c \cdot \log \log n \cdot \log \log \log \log n}{(\log \log \log n)^{2}}$ infinitely often. Back in 1936, Erdős [5] had established a similar result but without the loglogloglogn factor in the numerator. The Erdős US $\$ 10,000$ problem is to prove or disprove that the constant $c$ can be chosen arbitrarily large. See "Note Added in Proof" 
in this article. Ron Graham, who was the financial caretaker of Erdős for many years, has a pot of money left over to give out the prizes when the problems are solved.

Among Erdôs's many fundamental contributions it is universally agreed that the two most important are (i) his joint paper with Mark Kac [9], which ushered in the subject of probabilistic number theory, and (ii) his elementary proof of the prime number theorem along with Atle Selberg.

The first proof of the prime number theorem was given toward the end of the nineteenth century simultaneously by Hadamard and de la Vallee Poussin by utilizing the properties of the zeta function $\zeta(s)$ as a function of the complex variable $s$ as envisioned by Riemann. The method of this proof shows that the prime number theorem is equivalent to the assertion that $\zeta(1+i t) \neq 0$ for real $t$. This led to the belief that any proof of the prime number theorem had to rely on the theory of functions of a complex variable. The noted British mathematician G. H. Hardy challenged the world to produce an "elementary" proof of the prime number theorem, namely, a proof that uses only properties of real numbers. He proclaimed that if such an elementary proof is found, then the books would have to be rewritten, since it would change our view of how the subject hangs together. In 1949 Erdős and Selberg created a sensation by producing such an elementary proof. The elementary proof was actually found by them jointly by starting with a fundamental lemma of Selberg, but for various reasons (that we shall not get into here) they had a misunderstanding and decided to write separate papers. Selberg's paper [19] appeared in the Annals of Mathematics; Erdős published his paper [6] in the Proceedings of the National Academy of Sciences.

In contrast, the Erdős-Kac collaboration [9] was a happy story. To understand this in context, we mention that in 1917 Hardy and Ramanujan [13], who did the first serious investigation of $v(n)$, the number of prime factors of $n$, showed that the average order of $v(n)$ is asymptotically $\log \log n$. They noted that $v(n)$ also has normal order $\log \log n$. This means that, for every $\epsilon>0$, $v(n) / \log \log n$ is almost always between $1-\epsilon$ and $1+\epsilon$. They also showed that it makes no difference whether the prime factors of $n$ are counted distinctly or with multiplicity. In 1934, Paul Turán, another great Hungarian mathematician who was Erdős's close friend, gave a simpler proof [21] of the Hardy-Ramanujan results by computing an upper bound for the second moment of $v(n)$ with mean $\log \log n$ and noted that a similar second moment estimate could be given for certain additive functions, namely, functions $f(n)$ which like $v(n)$ satisfy $f(m n)=f(m)+f(n)$ when $m$

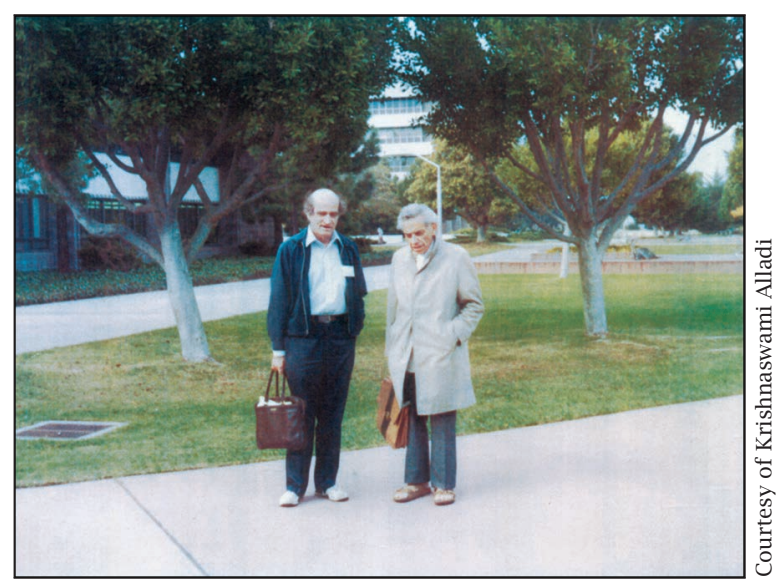

Paul Erdős and Ernst Straus on the campus of UC Santa Barbara during the West Coast Number Theory Conference, December 1978.

and $n$ are relatively prime. The Hardy-Ramanujan and Turán results gave a hint of probabilistic underpinnings, but this became clear only later. In 1939 the great probabilist Mark Kac was giving a lecture at Princeton outlining various applications of probability. He constructed a model for the study of additive functions and conjectured that the distribution of a wide class of additive functions about their average order and with an appropriately defined variance would be Gaussian. Erdős, who was in the audience, perked up. He realized that Kac's conjecture could be proved using the Brun sieve, a topic in which Erdős was a master. He spoke to Kac after the lecture and they proved the conjecture together. To quote Erdős (see [7]): "Neither of us completely understood what the other was doing, but we realized that our joint effort will give the theorem, and to be a little impudent and conceited, probabilistic number theory was born! This collaboration is a good example to show that two brains can be better than one, since neither of us could have done the work alone." Subsequently Kubilius [15] extended the ideas and methods of Turán and of Erdős-Kac to treat the non-Gaussian cases as well. The subject of probabilistic number theory, ushered in by Erdős and Kac, is an active field of research today (see the two-volume book of Elliott [3]).

Another significant example of a paper of Erdős that led to a major field of study is his work with Rényi on random graphs [10]. There are numerous other ideas of Erdős that have had, and continue to have, widespread influence. The articles that follow describe many developments whose origins can be traced back to Erdős.

Many great mathematicians have written papers that have influenced the development of the subject and created new fields of study. What sets Erdôs apart from all these luminaries is the 


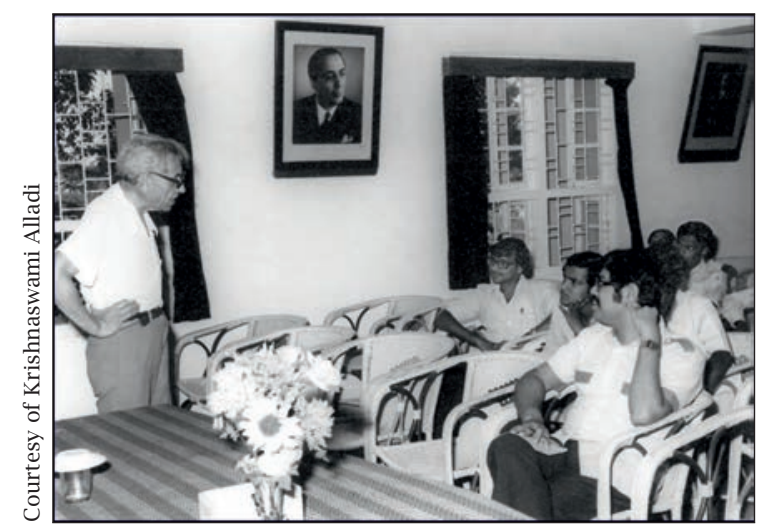

Paul Erdős lecturing at the Institute of Mathematical Sciences in Madras, India, in January 1984. Krishna Alladi is in the audience in the first row.

manner in which he collaborated with hundreds of mathematicians junior and senior and profoundly influenced them. It was his life's mission to seek out talented youngsters and mentor them in the study of mathematics. He jokingly said that he sought young collaborators, because he believed that he would be alive if at least half his collaborators were alive! The two of us will briefly share our experience in collaborating with Erdős.

\section{Alladi's First Meeting and Collaboration with Erdős}

As an undergraduate student in Madras, India, I was working on number theory on my own and read several introductory books. I noticed that there was practically nothing in these books on the sum of the prime factors of an integer. So I defined $A(n)$ to be the sum of the prime factors of an integer (summed with multiplicity) and observed it had two very nice properties: (i) $A(m n)=A(m)+A(n)$, for all $m, n$, and (ii) the number of solutions to $A(n)=m$ is the number of partitions of $m$ into primes. Thus I was convinced that $A(n)$ was worthy of a closer study, and I investigated many of its properties. I was advised that I should contact Erdős to evaluate this work. Thus I sent a letter to the Hungarian Academy of Sciences, requesting that it be forwarded to Erdős. Within three weeks I received a response from him saying that soon he would be at the Indian Statistical Institute in Calcutta in December 1974 to speak at a conference for the 100th birth anniversary of its founder, Professor Mahalanobis. He enquired whether I could come to Calcutta. I responded saying that my paper on this topic was accepted for presentation at that conference, but I could not go due to my university exams. I said that my father, who will be giving one of the main lectures on probability at that conference, will be presenting my work in a special session talk. Erdós attended my father's presentation of my work. He went up to him and said, "While I am pleased to meet you, I would be happier to meet your son." He then told my father that he was scheduled to fly to Sydney from Calcutta a week later, but he was willing to reroute his journey, fly via Madras to Sydney to meet me. This gesture by a great mathematician to journey out of the way to meet a student speaks volumes about his passion to spot and encourage young mathematicians.

I went to the Madras airport to meet Erdős. Needless to say I was very nervous. He put me at ease by saying hello and immediately afterward the following: "Do you know my poem about Madras:

This to the city of Madras

the home of the curry and the dhal, where Iyers speak only to Iyengars, and Iyengars speak only to God"?

I said I did not know this poem. He said this is modeled along a similar well-known poem about Boston:

This to the city of Boston

the home of the bean and the cod, where Lowells speak only to Cabots, and Cabots speak only to God.

The Iyers and Iyengars are two Brahmin castes of the Hindu religion. The great Nobel laureate physicist Sir C. V. Raman was an Iyer, and Srinivasa Ramanujan was an Iyengar.

He spent three days in Madras, and I was with him all the time. When I told him that I had calculated the average order of $A(n)$ to be asymptotically $\pi^{2} n / 6 \log n$ but could not determine its normal order, he said that $A(n)$ will not have a normal order because $A(n)$ is dominated by $P_{1}(n)$, the largest prime factor of $n$, and $P_{1}(n)$ does not have a normal order. Then I asked him if $P_{1}(n)$ is subtracted from $A(n)$, would the remaining sum be dominated by $P_{2}(n)$, the second largest prime factor on $n$, and so on? He said that this is very likely and that my question was very nice. So this led to our very first paper [2], in which we showed among other things that if $P_{k}(n)$ denotes the $k$ th largest prime factor of $n$, then $A(n)-P_{1}(n)-\cdots-P_{k-1}(n)$ and $P_{k}(n)$ have the same average (asymptotically), namely, $c_{k} n^{1 / k} / \log ^{k} n$, where $c_{k}$ is a rational multiple of $\zeta(1+1 / k)$. All in all, I wrote five joint papers with him.

In December 1974 I had applied for admission to graduate schools in America. Erdős said he would write exactly one letter for me regarding my graduate admission. So while in Madras, he wrote a letter to Ernst Straus, his long-time friend at UCLA. By the end of January 1975, I received a Chancellors Fellowship from UCLA for my PhD, and I went there to work under Straus. Thus 
my contact with Erdôs profoundly influenced my career. He corresponded with me from 1975 until his death in 1996 and supported my mathematical and professional progress. Over the years my family and I had the pleasure of hosting him in India, Florida, and in almost every place where I went for an extended visit.

\section{Krantz's Collaboration with Erdôs}

In the early 1980s I had a big fight with my colleague Tory Parsons. We were very fond of each other and did not really want to fight, so we ended up kissing and making up. We did so by talking about mathematics. And we ended up writing a little paper about a covering lemma. The following summer, Tory gave a talk on our paper at a conference in Europe. In the middle of the talk Erdős jumped up and shouted, “But don't you realize that your result will allow us to prove the following theorems?" And Chris Godsil jumped up and said, "And you can also prove these other theorems." Next thing we knew we had a four-way paper going [8], and three of us earned an Erdős number of one. This just goes to show that even fighting can have fruitful results.

\section{Honoring the Legacy of Erdós}

In the last quarter-century, books and movies on the remarkable life of Paul Erdős have been produced. The most well-known book is by Paul Hoffman, entitled The Man Who Loved Only Numbers [14], and it appeared in 1998 after Erdős passed away. The equally charming book entitled My Brain Is Open by Bruce Schechter [18] also appeared in 1998. Erdős loved to discuss mathematics with almost anyone, and whenever he was ready for discussion, he would say, "Go ahead, my brain is open." Hence the title of the second book. The story of Erdős rerouting his travel to meet Alladi is described in this book in the opening chapter, entitled "Traveling", thanks to Ron Graham, who drew the attention of the author, Bruce Schechter, to this story. Both of these books are for the general public and convey the greatness of Erdôs as a man and as a mathematician.

To complement these books is a nice documentary film called $n$ Is a Number by Paul Csicsery, which has been shown at several major conferences. Many top mathematicians who worked closely with Erdós and collaborated with him are interviewed, and there are many lovely clips of Erdős in discussion with mathematicians around the world.

For Erdős's seventieth birth anniversary, Combinatorica, a journal he founded, brought out a special volume in his honor. Three years after Erdős passed away, a conference entitled "Paul Erdős and his mathematics" was held at the Hungarian Academy of Sciences, Budapest, from July 4 to 11, 1999. A two-volume book [12] under the same title was published comprised of mathematical papers and reminiscences by several of the main speakers at that conference. More than five hundred mathematicians attended that conference, and some of them contributed papers to the memorial volume of Combinatorica.

In 1999 the American Mathematical Society started the Erdős Memorial Lectures with support from a fund created by Mr. Beal, a Dallas banker and mathematics enthusiast. This lecture is delivered annually at one of its meetings. A year earlier, in 1998, at the University of Florida, where Erdôs visited every spring, the annual Erdős Colloquium was launched during Alladi's term as chair. Ron Graham gave the first Erdős Colloquium in Florida as well as the first Erdős Memorial Lecture for the AMS. One year earlier, in 1997, Memphis State University (where Erdős was an adjunct professor since 1975) launched the Erdős Memorial Lectures, the first of which was delivered by Vera T. Sós.

Erdős, like Ramanujan, was such an unusual personality that articles about him have appeared in magazines such as the New Yorker, Discover, and the like. When he died, both the New York Times and the London Times published substantial obituaries.

\section{Awards and Distinctions}

Erdős received many prizes and much recognition for his monumental contributions. We mention just a few. He was awarded the 1951 Cole Prize of the AMS for his many fundamental papers and specifically for his "Elementary proof of the prime number theorem". In 1983 he was the recipient of the Wolf Prize for his lifelong contributions. He was also given an Honorary Doctorate by Cambridge University in 1991. He was elected a Member of the US National Academy of Sciences and also a Foreign Member of the Royal Society. He was elected as a Member of the Hungarian Academy of Sciences in 1956. But these laurels rested lightly on his shoulders. He always gave away the prize money he received for a good mathematical cause. When interviewed for the documentary $n$ Is a Number, Erdős said that he would trade all his awards for a nice theorem and its proof. To him what was important was to prove and conjecture.

An academician must be judged not only by the quality and significance of his contributions but also by the work of his direct disciples and many others he influenced through his ideas. In 1998 Tim Gowers of Cambridge University was awarded the Fields Medal. Gowers was in a sense a grand student of Erdôs, because Gowers received his $\mathrm{PhD}$ under the direction of Bela Bollobás, who was a disciple 


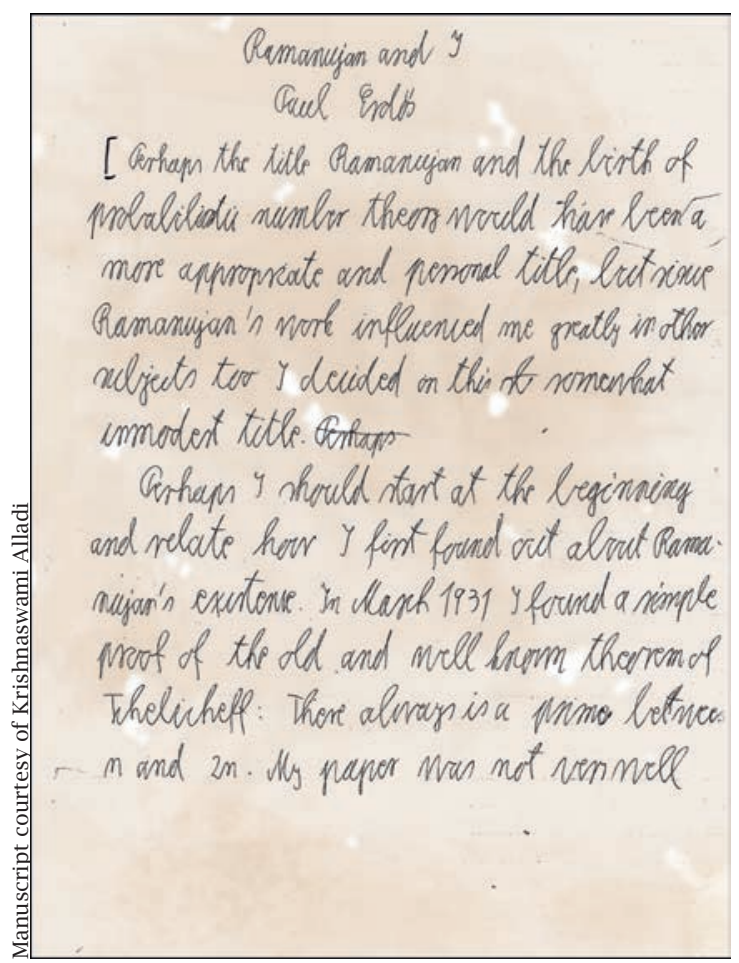

First page of the handwritten manuscript "Ramanujan and I" by Erdős, which he wrote for the Ramanujan Centennial.

of Erdős. The Fields Medal to Gowers was a major recognition by the international mathematical community of the importance of combinatorial methods and the kind of mathematics that Erdős pursued. This was followed by the 2006 Fields Medal to Terence Tao for contributions to many areas of mathematics, especially to the field of additive combinatorics. The award of the 2012 Abel Prize to E. Szemerédi, a protege of Erdős, is yet another recognition for Erdős-type mathematics and for the Hungarian mathematical tradition. Even though Erdős started out in number theory and made several pioneering contributions to that area, in later years his attention was mostly directed towards combinatorics, graph theory, and discrete mathematics, and he strongly influenced the development of these fields. If Erdós were alive today, he would be the happiest person to see that combinatorics and discrete mathematics have been given their due place in mathematics.

\section{References}

[1] M. Aigner and G. M. Zeigler, Proofs from The Book, Springer, Berlin, 1998.

[2] K. Alladi and P. ERDós, On an additive arithmetic function, Pacific J. Math. 71 (1977), 275-294.

[3] P. D. T. A. ElliotT, Probabilistic Number Theory. I, II, Springer, New York, 1980.

[4] P. ERDős, Beweis eines Satzes von Tscebyschef, Acta Sci. Math. (Szeged) 5 (1930-1932), 194-198.
[5] - On the difference between consecutive primes, Quart. J. Math., Oxford Ser., 6 (1935), 124-128.

[6] , On a new method in elementary number theory which leads to an elementary proof of the prime number theorem, Proc. Nat'l. Acad. Sci. USA 35 (1949), 374-384.

[7] , Ramanujan and I, in Number Theory, Madras 1987, Proc. Ramanujan Centenary Conf. (K. Alladi, ed.), Springer Lecture Notes in Math.,1395, 1989, pp. $1-20$.

[8] P. ERdős, C. Godsil, S. G. Krantz, and T. D. PARsons, Intersection graphs for families of balls in $R^{n}$, Eur. $J$. Combin. 9 (1998), 501-505.

[9] P. ERDÖS and M. KAC, The Gaussian law of errors in the theory of additive number theoretic functions, Amer. J. Math. 62 (1940), 738-742.

[10] P. ERDŐs and A. RÉNYI, On the evolution of random graphs, Magyar Tud. Akad. Mat. Kutató Int. Kozl. 5 (1960), 17-61.

[11] B. GREEN and T. TAO, The primes contain arbitrarily long arithmetic progressions, Annals of Math. (2) 167 (2008), 481-547.

[12] G. HALASZ, L. LovÁsz, M. Simonovits, and V. T. Sós, eds., Paul Erdós and his mathematics. I, II, Bolyai Society Math. Studies, 11, Springer, Berlin, 2002.

[13] G. H. HARDY and S. RAMANUJAN, On the normal number of prime factors of a positive integer $n$, Quart. $J$. Math. (Oxford) 48 (1917), 76-92.

[14] P. Hoffman, The Man Who Loved Only Numbers, Hyperion, New York, 1998.

[15] J. KubILIUS, Probabilistic methods in the theory of numbers, Uspehi Math. Nauk. 11 (1956), 31-66.

[16] L. LovÁsz, I. Z. RuZSA, and V. T. Sós, eds., Erdös Centennial, Bolyai Society Math. Studies, 25, Springer, Berlin, 2013.

[17] R. A. RANKIn, The difference between consecutive prime numbers, J. London Math. Soc. 13 (1938), 242247.

[18] B. SCHechter, My Brain Is Open-The Mathematical Journeys of Paul Erdös, Simon and Schuster, New York, 1998.

[19] A. SELBERG, An elementary proof of the prime number theorem, Annals of Math. (2) 50 (1949), 305-313.

[20] E. SZEMERÉDI, On sets of integers containing no $k$ elements in arithmetic progression, Acta Arith. 27 (1975), 199-245.

[21] P. TURÁN, On a theorem of Hardy and Ramanujan, J. London Math. Soc. 9 (1934), 274-276.

[22] E. Westzynthius, Über die Verteilung der Zahlen, die zu den n ersten Primzahlen teilerfremd sind, Comm. Phys. Math. Helsingfors 25 (1931), 1-37.

[23] Y. ZHANG, Bounded gaps between primes, Annals of Math. (2) 179 (2014), 1121-1174.

\section{Note Added in Proof}

Very recently there has been tremendous progress on both the small gap and large gap problems. Maynard [25] has stunned the world by showing that the gap between primes can be made made $\leq 600$ infinitely often. Following Zhang's bounded gap theorem, the Polymath Project led by Tao had achieved a bound of 4,680 vastly improving Zhang's bound of seventy million, but Maynard has reduced this even further. Maynard is awarded the 2014 
SASTRA Ramanujan Prize for this achievement and other results. See [27] for the latest results on the bounded gap problem. In the last few months, Ford-Green-Konyagin-Tao [24] and Maynard [26] have announced a solution to the Erdôs $\$ 10,000$ problem by showing that the constant in Rankin's lower bound can be made arbitrarily large. The methods in [24] and [26] are different.

\section{References}

[24] K. Ford, B. Green, S. Konyagin and T. TAO, Large gaps between consecutive prime numbers, preprint, http://arxiv.org/abs/1408.4505.

[25] J. MAYNARD, Small gaps between primes, Annals of Math. (to appear).

[26] __ Large gaps between primes, arXiv1409.5110v1[math.NT].

[27] D. H. J., Polymath: The "Bounded Gaps Between Primes" Polymath Project, Newsletter EMS 94 (2014), 13-23.

\section{László Lovász and Vera T. Sós}

\section{Erdős Centennial}

It would be impossible to discuss the tremendous work of Paul Erdôs especially in such a short article. All we can do is to contribute some impressions and ideas about the nature of his work, flavored by some quotations from letters of Erdôs and by a few personal impressions and experiences. Several "mathematical," and not only mathematical, biographies of Erdôs were written, among these we mention here two thorough ones written by Babai [2] and Bollobás [6]. His work was treated in depth in a number of volumes containing expert articles [20], [21], [22], [24], and even on the pages of these Notices [4].

The idea of the present issue of the Notices arose in connection with the Erdós Centennial Conference we organized in summer 2013. To be precise, we organized three Paul Erdős conferences in Budapest: The first took place in 1996, one day after his funeral. At that one-day meeting, our goal was to give an immediate short survey of his oeuvre, a demonstration of his unique role in mathematics in the past seven decades. The second conference took place three years later, in 1999, when our primary aim was to cover as much as possible the full scope and richness of his mathematics and its impact. The third conference was in 2013 to celebrate the hundredth anniversary of his birth. The intention of this third one was to give a panorama of the monumental development originating in his mathematics, of the wide-ranging

László Lovász is professor of computer science at Eötvös Lorand University. His email address is 1ovasz@cs . e1 te. hu.

Vera T. Sós is professor of mathematics at the Alfred Rényi Institute of Mathematics, The Hungarian Academy of Sciences. Her email address is sos@renyi . hu.

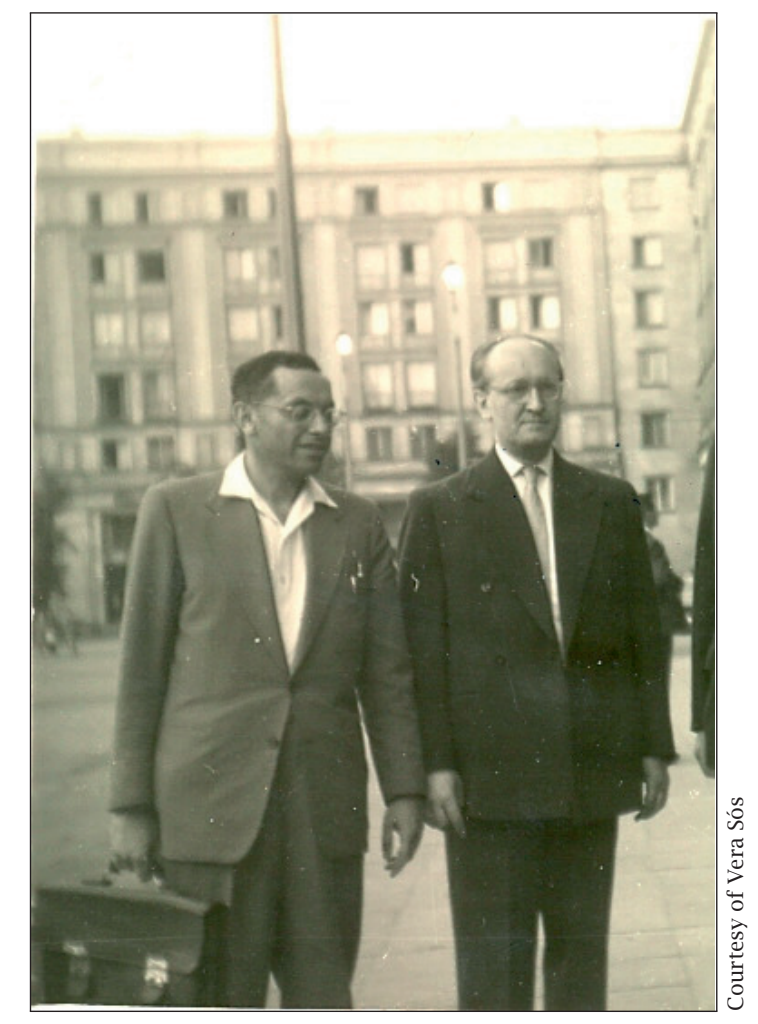

Paul Erdős and Paul Turán were great mathematicians, close friends, and partners in several important collaborations.

influence of his work, and to give some indication of possible trends in the future. The success of the conference surpassed all our expectations: more than twice as many mathematicians participated as we first expected, illustrating the tremendous interest in his work and its proceeds.

Trying to collect a few thoughts about the character of Erdős's mathematics, our starting point could be what he wrote in a letter that included a scientific biography written by him in the late 1970s:

To finish this short outline of my scientific biography, I observe that most of my papers contain some type of combinatorial reasoning and most of them contain unsolved problems.

Indeed, a special trait all across his work was his unparalleled power of formulating and posing problems and conjectures. He had a special sense for asking just the right questions: how else can we explain that many of his innocent-looking problems have opened up new areas, in some cases after several decades? He wrote the first "problem paper" in 1956 [9], which contained six problems. After more than half a century, in spite of the many important results and methods initiated by this paper, none of these six problems is completely solved. 
In one of his letters from 1979 he wrote:

I am writing a paper with the title: 'Combinatorial problems I would most like to see solved'-the subjective title is better because then I do not have to write about my opinion on the importance of the problems.

While Erdős often just asked a problem in a very compact form without mentioning any reasons, these problems were not as spontaneous as it would seem. An example: In a letter to Paul Turán in 1938 he formulated a conjecture about the maximum number of $k$-element subsets of an $n$ element set with the property that any two of them intersect in at least $r$ points. He mentioned that with the help of Ko he could prove the case $r=1$, and he added the remark: "The theorem could have beautiful applications in number theory." The proof was published only in 1961 in a famous paper by Erdós, Ko, and Rado [15], but possible applications in number theory are not mentioned in the paper.

In its simplest form, the Erdôs-Ko-Rado Theorem says that to get the largest number of $k$-subsets of an $n$-set $(n \geq 2 k)$ that mutually intersect, one should take all $k$-subsets containing a given element. When reading such a statement, one realizes that many similar problems can be raised about subsets of a finite set, and then, depending on one's temperament, one might escape, or one might be challenged by, the fact that such basic questions are unsolved. Luckily, Erdós and several others felt the challenge, and over a relatively short period a wealth of basic questions in extremal set theory were answered. The theorems of Sperner (about sets not containing each other), Erdős-de Bruijn (about sets, any two intersecting in exactly one element), Erdős-Rado (about sets among which no three mutually have the same intersection) and Kruskal-Katona (about $k$-sets covering the least number of $r$-sets) are not only standard theorems in combinatorics textbooks, but they have very important applications in geometry, number theory, computer science, and elsewhere. These problems, which arise in a very simple and natural way, are often quite difficult to solve, and in some cases a complete solution is still missing after decades of intensive research.

Another characteristic of his mathematics was that very often his questions and proofs reveal deep relationships between different areas in mathematics. Even though he was never directly involved in computer science, he had an essential influence on it, mostly through extremal set theory and the probabilistic method. These connections could not have been foreseen, except perhaps by Erdoss himself. (About this aspect of his work see Babai [3].) Using his own words from the late seventies:
I am basically a pure mathematician and had little contact with applied mathematics, I expect that my paper with Rényi on the evolution of random graphs will be used in several branches in science-Rényi planned to work in this direction but was prevented by his untimely death. Graham, Szemerédi and I [16] have a paper on problems raised by computer scientist but I am not competent enough to judge their importance for applications.

Erdős started out as a number theorist, and number theory remained present in his mathematics all the time. There are several survey articles dealing with his work on the theory of primes, equidistribution, diophantine approximation, additive and multiplicative number theory, and many more. Discovering the combinatorial nature of some of his early number theory problems led him to general questions in combinatorics and in graph theory. He writes in his above-quoted "scientific biography":

My main subjects are: number theory (a subject which interested me since early childhood when I learned from my father Euclid's proof that the number of primes is infinite), combinatorial analysis, set theory, probability, geometry and various branches of analysis.

His work in set theory often arose from combinatorics as infinite versions of finite problems. His problems and results in geometry and algebra also have a combinatorial flavor. He was the driving force behind the development of large areas of modern combinatorics, including extremal graph theory and extremal set theory. Since combinatorics is the best-known area of his work (which is due, at least in part, to the fact that this was the focus of his work in his later years), we will not go into the details of these results.

Another area that Erdős introduced into several branches of mathematics is probability. The interaction with probability is a very hot topic in number theory, combinatorics, computer science, and other areas, and the pioneering work of Erdös is present all over this work. We could talk about four different ways in which he contributed to this field.

1. He studied problems in pure probability theory (often with a combinatorial flavor but belonging to mainstream probability), like random walks or the Law of Iterated Logarithm. As to this last work, let us quote Bollobás [6]: "There are very few people who have contributed more to the fundamental theorems in probability theory than Paul Erdős." 
2. Starting with problems in number theory, he showed how to exploit the random-like behavior of different structures. Let's quote his own words [13]:

Heuristic probability arguments can often be used to make plausible but often hopeless conjectures on primes and on other branches of number theory.

The deliberate and systematic application of probability theory to number theory started with the celebrated Erdős-Kac theorem [14]. For a detailed review of the story of this theorem, see the article of Alladi-Krantz in this issue of the Notices. Erdős himself wrote about the formation of the Erdôs-Kac theorem several times; let's quote from [10]:

I conjectured that the convergence of the three series is both necessary and sufficient for the existence of the distribution function (of an additive function) but this I could not prove due to my gaps of knowledge in Probability Theory....After the lecture (of Kac) we got together... and thus with a little impudence we would say, that probabilistic number theory was born.

Elliott writes in his book [8] about this theorem:

This result, of immediate appeal, was the archetype of many results to follow. It firmly established the application of the theory of probability to the study of fairly wide class of additive and multiplicative functions.

3. Perhaps most important of Erdős's achievements is the "probabilistic method," the use of probability to prove the existence of certain objects without explicitly constructing them (and whose explicit construction is sometimes still open sixty years later). This issue of the Notices contains other papers that describe this fundamental method and its applications, and we can also refer to the books of Alon and Spencer [1] and Erdős and Spencer [19].

4. The Erdős-Rényi theory of random graphs is the first major example of the investigation of random structures. To be precise, random sets of integers, random polynomials, random matrices, and other random structures were considered before by several mathematicians (including Erdős and Rényi themselves), but random graphs were the first where a comprehensive theory arose that showed how basic properties of these graphs are different from their deterministic counterparts. Several books have been written about random graphs [5], [23]. The Erdős-Rényi random graphs serve as basic examples in the recent explosion of random graph models for many real-life networks (like the Internet and social networks), where the understanding and explanation of the differences from this basic model is the main goal.

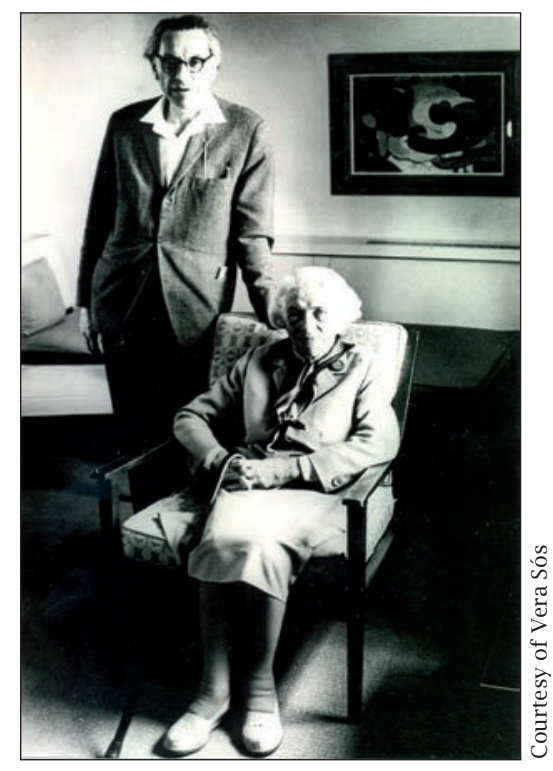

Paul Erdős with his mother, who travelled with him around the world until her death in 1971 .

Analysis, in particular approximation, interpolation, polynomials, complex functions, and infinite series, were also in the foreground of his research from the thirties through the sixties. His analytic power can be felt in his papers all along. It is best to quote Paul Turán, who was an early collaborator of Erdős and wrote a detailed survey on Erdős's work on the occasion of his fiftieth birthday [27]. (This became an important source for many later articles on Erdős.) Out of the several topics in analysis which Turán discussed in this paper, let's quote what he wrote about the application of probability in analysis:

"The application of probabilistic methods runs right through the whole oeuvre of Erdős and this holds for his works in analysis as well. In this connection I have in my mind especially three of his papers, the first of which was published in 1956 in the Proc. London Math. Soc. with Offord [17], the second in 1959 in the Michigan Math. J. with Dvoretzky [7], the third will be published with Rényi in the volume to be issued to celebrate the 75th birthday of György Pólya [18]. In the first they showed that if $\varepsilon_{v}= \pm 1$, then the $2^{n}$ equations

$$
1+\varepsilon_{1} x+\cdots+\varepsilon_{n} x^{n}=0
$$

have, with at most $o\left(2^{n} / \sqrt{\log \log n}\right)$ exceptions,

$$
\frac{2}{\pi} \log n+o\left(\log ^{\frac{2}{3}} n \log \log n\right)
$$

real roots each.

"The second gives an existence proof of the nice theorem that there exists a power series $\sum_{0}^{\infty} \frac{e^{i \alpha_{n}}}{\sqrt{n}} z^{n}$ with real $\alpha_{n}$ that diverges on the whole unit circle (that this can be achieved excluding a set 


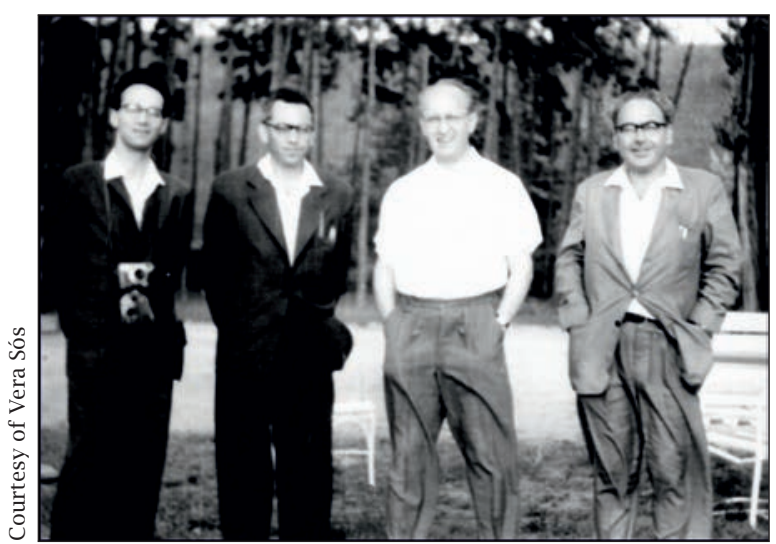

(From I to r) George Graetzer, Paul Erdős, Paul Turán and Alfred Renyi.

of measure zero was known). In the third they solve an old problem of Zygmund in connection with a theorem of N. Wiener. This theorem of Wiener states (in a weakened form) that if the series

$$
\sum_{v}\left(a_{v} \cos l_{v} x+b_{v} \sin l_{v} x\right)
$$

where the $l_{v}$ 's are positive integers satisfying

$$
\lim _{v \rightarrow \infty}\left(l_{v+1}-l_{v}\right)=\infty
$$

is Abel-summable in an arbitrarily small interval $(a, b)$ to a function $f(x)$ belonging to $L_{2}$, when we have $\sum\left(a_{v}^{2}+b_{v}^{2}\right)<\infty$, hence the series is the Fourier series of a function belonging to $L_{2}$ on, the whole $[0,2 \pi]$, thus $f(x)$ has an extension to $[0,2 \pi]$ that is in $L_{2}$ and whose Fourier series is the given series. Ingham, Zygmund and Marcinkiewicz and the author of these lines gave much simpler proofs of this theorem than the original; some twenty years ago Zygmund raised the question whether the theorem can be extended to a class $L_{q}$ with $q>2$ in the place of $L_{2}$. Now Erdős and Rényi with probabilistic methods showed for every $q>2$ the existence of a trigonometric series satisfying the above lacunarity condition that is summable to a function continuous in $(a, b)$ for every $0<a<b<2 \pi$ and still the series is not the Fourier series of any function belonging to $L_{q}$ on $[0,2 \pi] . "$

Erdôs was always very supportive of young people. In the 1960s, when the Cold War began to melt and he started to spend more time in Budapest, he would often sit in the lobby of his hotel all day, with students and young researchers coming and going, discussing their new results, and learning about new developments and new problems from all over the world. One of us (the first author) was lucky enough, as a high school student, to have the opportunity to stay there and take part in these discussions. The effect of these discussions on how to look at mathematics, research, colleagues, science, and the world has lasted a lifetime.

From this experience, and in general from the attitude of Erdős towards open problems, conjectures, dissemination of ideas and collaboration, his basic (probably unstated) philosophy can be distilled: he believed in total openness in research, where the goal is to advance knowledge, and we all work together to achieve it.

Let me (the second author) also mention my first and last meeting with Paul Erdős-the beginning and the end of almost fifty years of acquaintance and more than three decades of collaboration, partly in several hundreds of letters. I met Erdős the first time in 1948, when he returned to Hungary after a break of ten years. My high school teacher, Tibor Gallai, one of Erdős's best friends, introduced me to him. I cannot recall the particulars of our conversation, but I am sure he asked mathematical questions, as he usually did when meeting young people interested in mathematics. However, I remember that because of a long break his visit had a special significance. Let me say a few words about this.

Erdős and Gallai were members of the now legendary "Anonymus group."1 The members of this group met regularly during their university years at the Statue of Anonymus in City Park in Budapest. Lifelong friendships were formed between them, and their meetings had a deep impact on their professional lives as well.

Arranged by Mordell, Erdős spent the years 1934-38 in Manchester. During this period he returned to Hungary quite regularly three times a year for shorter visits. In 1938 he decided to leave Hungary, with its adverse and deteriorating political situation. He had to leave his family, he had to leave his friends. Then came the war years; Erdős returned to Budapest only ten years later to see his mother and his friends. This was the occasion when, in December 1948, I met Erdôs for the first time.

In September 1996 we both attended a graph theory conference in Warsaw. Our plan was to go from Warsaw, together with András Sárközy, to Vilnius to participate in a number theory conference the following week. On the morning of Wednesday, September 18, he gave his very last problem lecture. The last problem he mentioned was a problem of Hajnal (and perhaps himself). He got stuck, started again, and this was repeated two

\footnotetext{
${ }^{1}$ László Alpár (1914-1991), Pál Erdôs (1913-1996), János Erôds, (1912-1944), Ervin Feldheim (1912-1944), Géza Grünwald (1913-1944), Tibor Grünwald (Gallai) (19121992), Eszter Klein (1910-1975), Dezső Lázár (1913-1943), György Szekeres (1911-1975), Pál Turán (1910-1976), Márta Wachsberger (Sved) (1911-2005), Endre Weiszfeld (1913-1976).
} 
more times. After the third attempt, he put down the chalk and finished the talk. The audience broke out in applause, and he responded, "Thank you. I know this is meant as a consolation!" There was an excursion the same afternoon, which he skipped, partly because of the cold weather. Instead of that, the rest of the day became the last hours we spent together, switching between topics and problems perhaps more often than at other times. Paul Erdős passed away on Friday, September 20 [26].

Erdős's brilliant mathematical thinking, pure character, helpful and sympathizing nature; his quest for truth in science, politics, everyday lifethese are what motivated his untiring, relentless activity and creativity until his last days. His personality is perhaps evoked by the simple lines he wrote one morning in 1976:

It is six in the morning, the house is still asleep, I am listening to lovely music, while writing and conjecturing. ${ }^{2}$

\section{References}

[1] N. Alon and J. SPENCER, The Probabilistic Method, Wiley-Interscience, 2000.

[2] L. BABAI, In and Out of Hungary, Paul Erdős, his friends, and times, in Combinatorics: Paul Erdös is Eighty, János Bolyai Mathematical Society, 1993.

[3] _ Paul Erdős (1913-1996). His influence on the theory of computing, Proc. 29th STOC, ACM Press, 1997, pp. 383-401.

[4] L. BABAi, C. Pomerance, and P. Vértesi, The mathematics of Paul Erdős, Notices of the AMS 45 (1998), 19-31.

[5] B. BollobÁs, Random Graphs, Academic Press, 1985.

[6] B. BollobÁs, To prove and conjecture: Paul Erdős and his mathematics, Amer. Math. Monthly 105 (1998), 209-237.

[7] A. DVORETZKY and P. ERDős, Divergence of random power series, Michigan Math. J. 6 (1959), 343-347.

[8] Peter D. T. A. ElliotT, Probabilistic Number Theory I-II, Grundlehren der Math. Wissenschaften, 239-240, Springer-Verlag, Berlin-New York, 1980.

[9] P. ERDős, Problems and results on additive number theory, in Colloque sur la Thèorie des Nombres, Bruxelles, 1955, George Thone, Liège; Masson and Cie, Paris, 1956, pp. 127-137.

[10] _ On some of my favourite theorems, in D. Miklós, V. T. Sós, T. Szőnyi (eds.), Combinatorics, Paul Erdős Is Eighty, Bolyai Society Mathematical Studies, 2, János Bolyai Mathematical Society, Budapest, 1996, pp. 97-132.

[11] , On the combinatorial problems which I would most like to see solved, Combinatorica 1 (1981), 25-42.

[12] _ On some of my problems in number theory I would most like to see solved, Number theory (Ootacamund, 1984) K. Alladi (ed.), Lecture Notes in Math., 1122, Springer, Berlin, 1985, pp. 74-84.

[13] _ Some applications of probability methods to number theory. Successes and limitations, in

${ }^{2}$ The original one, in Hungarian: "Reggel hat van s a ház még alszik, szép zenét hallgatok, s közben írok és sejtek.”

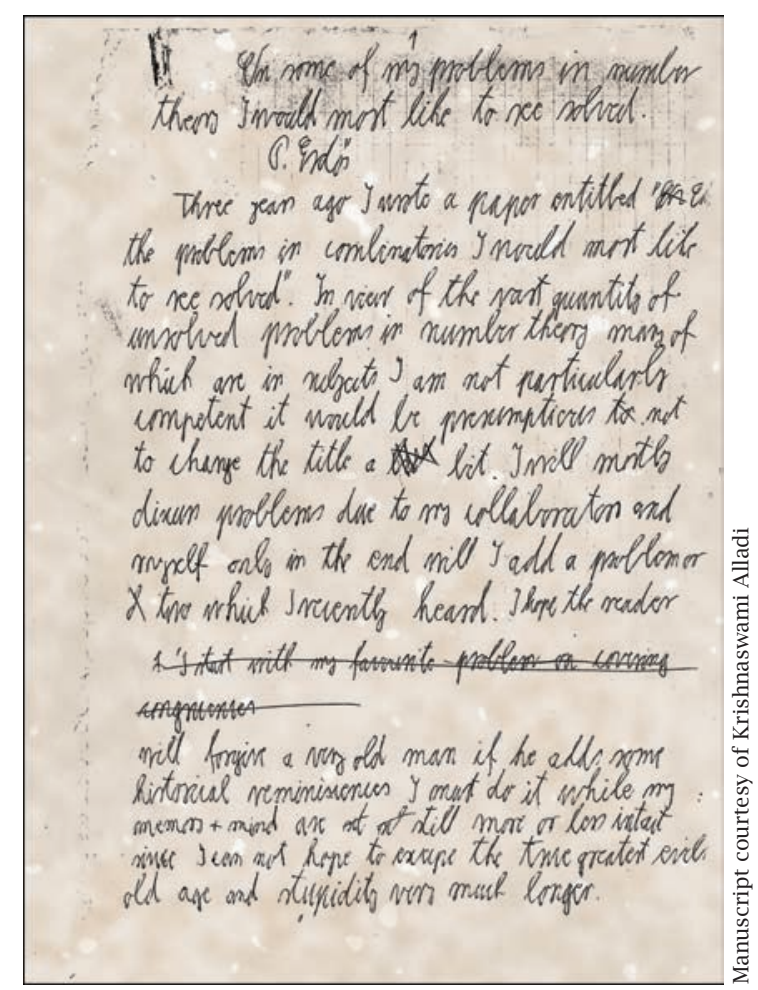

Erdös contribution to the Proceedings of the Conference on Number Theory in his honor for his 70th birthday held in Ootacamund, India in January 1984, and referred to in the Lovász-Sós article.

Capocelli, R. (ed.), Sequences (Naples-Positano, 1988), Springer, New York, 1990, pp. 182-194.

[14] P. ERDós and M. KAC, The Gaussian law of errors in the theory of additive number theoretic functions, Amer. J. Math. 62 (1940), 738-742

[15] P. ERDős, CHAO Ko, and R. RADO, Intersection theorems for systems of finite sets, Quart. J. Math., Oxford Ser. 12 (1961), 313-320.

[16] P. ERDŐS, R. L. GRAHAM, and E. SZEMERÉDI, On sparse graphs with dense long paths, Computers and Mathematics with Applications, Pergamon, Oxford, 1976, pp. 365-369.

[17] P. ERDős and A. C. OFFORD, On the number of real roots of a random algebraic equation, Proc. London Math. Soc. (3) 6 (1956), 139-160.

[18] P. ERDŐs and A. RÉNYI, On a problem of A. Zygmund, Studies in Mathematical Analysis and Related Topics, Stanford Univ. Press, Stanford, California, 1962, pp. 110-116.

[19] P. ERDős and J. SPENCER, Probabilistic methods in combinatorics, Probability and Mathematical Statistics, Academic Press, New York-London, 1974.

[20] R. L. GRAHAM and J. NEŠETřIL (eds.), The Mathematics of Paul Erdós. I-II, Springer-Verlag, New York, 1996.

[21] R. L. Graham, J. NeŠEtřIl, and S. Butler (eds.), The Mathematics of Paul Erdös. I-II (second edition), Springer-Verlag, New York, 2013.

[22] G. Halász, L. Lovász, M. Simonovits, and V. T. Sós (eds.), Paul Erdös and His Mathematics. I-II, Bolyai Society Mathematical Studies, 11, János Bolyai 
Mathematical Society, Budapest; Springer-Verlag, Berlin, (2002).

[23] S. Janson, T. Łuczak, and A. Ruczynski, Random Graphs, Wiley, 2000.

[24] L. LovÁsz, I. Z. RuzsA, and V. T. Sós (eds.), Erdós Centennial, Bolyai Society Mathematical Studies, 25, János Bolyai Mathematical Society, Budapest; SpringerVerlag, Berlin, 2013.

[25] V. T. Sós, Turbulent years: Erdős in his correspondence with Turán from 1934 to 1940, in Paul Erdös and His Mathematics, Bolyai Society Mathematical Studies, 11, János Bolyai Mathematical Society, Budapest; SpringerVerlag, Berlin, 2002, pp. 85-146.

[26]___ Paul Erdős, 1913-1996, Aequationes Math. 54 (1997), no. 3, 205-220.

[27] P. TuRÁn, Pál Erdôs is fifty, in Paul Erdós and His Mathematics, Bolyai Society Mathematical Studies, 11, János Bolyai Mathematical Society, Budapest; SpringerVerlag, Berlin, 2002, pp. 55-83. (Translation from Hungarian: Turán Pál, Erdős Pál 50 éves, Mat. Lapok 14 (1963), 1-28.)

\section{Ronald L. Graham and Joel Spencer}

Ramsey Theory and the Probabilistic Method Ramsey Theory was a lifelong interest of Paul Erdős. It began [11] in the winter of 1931-32. George Szekeres recalled:

We had a very close circle of young mathematicians, foremost among them Erdős, Turán and Gallai; friendships were forged which became the most lasting that I have ever known and which outlived the upheavals of the thirties, a vicious world war and our scattering to the four corners of the world. I $[\ldots]$ often joined the mathematicians at weekend excursions in the charming hill country around Budapest and (in the summer) at open air meetings on the benches of the city park.

Szekeres, Esther Klein, and Erdős attacked an unusual geometric problem: Is it true that for every $k$ there exists an $n$ so that given any $n$ points in the plane, no three collinear, some $k$ of them form a convex $k$-gon? Szekeres, in finding a proof of this conjecture, actually proved Ramsey's Theorem, which none of the three knew about at the time.

The mantra for Ramsey Theory is "Complete disorder is impossible." Let $s, r, k$ be positive integers. Then, Ramsey showed, for $n$ sufficiently large (dependent on $s, r, k$ ), the following holds: Let $\Omega$ have size $n$. Take any partition of the $s$-element

Ronald L. Graham is professor of computer science and engineering at the University of California at San Diego. His email address is graham@ucsd. edu.

Joel Spencer is professor of mathematics and computer science at New York University. His email address is spencer@cims.nyu.edu. subsets of $\Omega$ into $r$ colors. Then there will be a $k$ element set $S \subset \Omega$ which is monochromatic, in the sense that all of its $s$-element subsets are the same color. In the important special case $s=2$ one may think of an $r$-coloring of the edges of the complete graph $K_{n}$. While Erdős was not the originator of Ramsey Theory, he was its chief proponent, with conjectures and theorems in myriad directions that truly turned Ramsey's Theorem into Ramsey Theory.

A natural question arose: Just how big does $n$ need to be? We'll restrict here to $s=2$, though the other cases are also important. The Ramsey function $r(k)$ is the least $n$ such that if the edges of the complete graph $K_{n}$ are red/blue colored, then there will necessarily be a monochromatic $K_{k}$. The proof of Szekeres worked for $n=\left(\begin{array}{c}2 k-2 \\ k-1\end{array}\right)$ so that, thinking asymptotically, $r(k)<(4+o(1))^{k}$. In 1947 Erdős published a three-page paper [3] in the Bulletin of the AMS that had a profound effect on both the Probabilistic Method and on Ramsey Theory.

Theorem. Let $n, k$ satisfy

$$
\left(\begin{array}{l}
n \\
k
\end{array}\right) 2^{1-\left(\begin{array}{l}
k \\
2
\end{array}\right)}<1 .
$$

Then $r(k)>n$. That is, there exists a two-coloring of the edges of $K_{n}$ such that there is no monochromatic $K_{k}$.

Today, for those in the area, the proof is two words: Color Randomly! Consider a random coloring of the edges. For each of the $\left(\begin{array}{l}n \\ k\end{array}\right)$ sets $S$ of $k$ vertices there is a probability $2^{1-m}, m=\left(\begin{array}{l}k \\ 2\end{array}\right)$, that the $m$ edges are all colored the same. The probability of a disjunction is at most the sum of the probabilities, and so the disjunction has probability strictly less than one. Thus with positive probability the coloring is as desired. But (this part is sometimes called Erdős Magic) if there were no such coloring, then the probability would be zero, so, reversing, the coloring absolutely positively must exist.

Asymptotic analysis (from Erdős's paper) gives $r(k)>(\sqrt{2}+o(1))^{k}$. There have been some improvements in both the upper and lower bounds, most notably by David Conlon, but only for lowerorder terms. The gap between $\sqrt{2}$ and 4 has not moved since 1947 and is a central question in the field.

In 1950 [7], with Richard Rado, Erdős began the area of canonical Ramsey Theory. Let $S$ be an ordered set. They gave four special colorings of the pairs of $S$ : They could all have the same color; they could all have different colors; the color of $\{x, y\}$ with $x<y$ could be different for different $x$ and the same for the same $x$; the color of $\{x, y\}$ with $x<y$ 
could be different for different $y$ and the same for the same $y$. These they called the canonical colorings of the pairs of $S$. Fix $k$ and consider any coloring (no restriction on the number of colors) of the pairs of $\Omega$ with $\Omega=\{1, \ldots, n\}$. Then, for $n$ sufficiently large they showed that there must be a $k$-set $S \subset \Omega$ on which the coloring is canonical (there were similar results for triples, etc.).

Generalizing $r(k)$ the Ramsey function $r(l, k)$ is the least $n$ such that if the edges of the complete graph $K_{n}$ are red/blue colored, then there will necessarily be either a red $K_{l}$ or a blue $K_{k}$. The special case $l=3$ was a lifelong fascination of Erdős. Associating red/blue with whether or not an edge is in the graph, $r(3, k) \leq n$ means that every triangle-free graph on $n$ vertices must contain an independent set of size $k$. The argument of Szekeres from 1931-3 showed that $n=\left(\begin{array}{c}k+1 \\ 2\end{array}\right)$ has this property. In 1957 Erdős gave an intriguing geometric construction showing $R(3, k)>k^{1+c}$ for a small constant $c$. He returned [4] to the problem in 1961 with a probabilistic tour de force. He considered a random graph on $n$ vertices with adjacency probability $p=\epsilon n^{-1 / 2}, \epsilon$ a small constant. Such a graph $G$ will have many triangles. But then Erdôs ordered the edges of $G$ and considered them sequentially. He rejected an edge if it would form a triangle, thus tautologically forming a triangle-free subgraph $H$. Erdős then employed an array of probabilistic techniques that would be impressive even today, but that they were done so early is simply amazing. With them he showed that with high probability the graph $H$ would not have an independent set of size $k=c \sqrt{n} \ln n$. Reversing the variables, this gave $r(3, k)>c_{1} k^{2} \ln ^{-2} k$. For forty years, while alternate proofs of this result were given, the asymptotics of this lower bound were unchanged. In the meantime, Ajtai, Komlós, and Szemerédi improved the upper bound to $R(3, k)=O\left(k^{2} / \ln k\right)$. In 1995 [10] (with Peter Winkler and Steve Suen) Erdős returned once again to the lower bound. This time, rather than use an arbitrary ordering, they looked at a random ordering. They applied what we would call today a random greedy algorithm: $:^{3}$ the $\left(\begin{array}{l}n \\ 2\end{array}\right)$ potential edges were ordered randomly; an edge was rejected when it would form a triangle with previously accepted edges. Their analysis only improved the constant $c_{1}$ of Erdôs's 1961 paper. But it opened the door, and soon after Jeong Han Kim [12], using a minor modification of the random greedy algorithm, improved the lower bound to $r(3, k)=\Omega\left(k^{2} / \ln k\right)$, thus resolving the asymptotics of $r(3, k)$ up to a constant factor. Indeed, Tom Bohman in 2009 showed that Erdős's random greedy algorithm

\footnotetext{
${ }^{3}$ While Erdös's work has been tremendously influential in the analysis of algorithms, he himself never used that language.
}

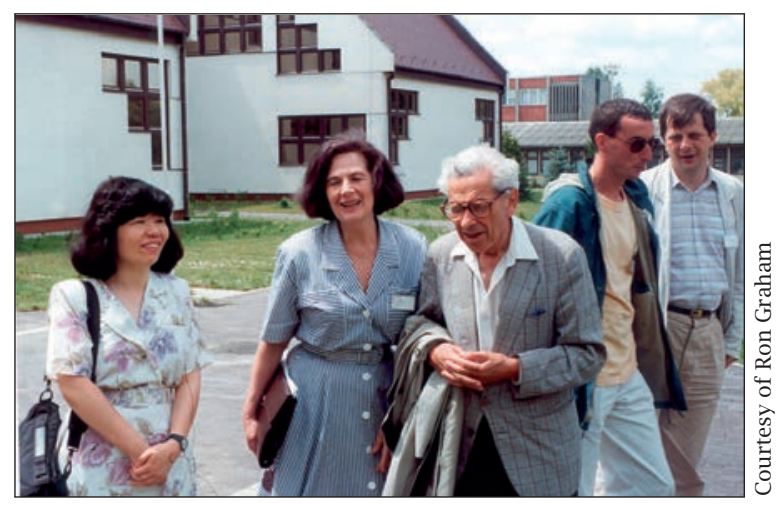

(From I to r) Fan Chung, Vera Sós, and Paul Erdős in Budapest.

did give Kim's result, although his proof involved elaborate modern methods. However, to illustrate our lack of knowledge here, the best current bounds on $r(4, n)$ are only

$$
\frac{c n^{\frac{5}{2}}}{\log ^{2} n}<r(4, n)<\frac{c^{\prime} n^{3}}{\log ^{2} n}
$$

for suitable positive constants $c, c^{\prime}$. In particular, we don't even know the correct exponent of $n$ !

In 1956 Erdős [8], with Richard Rado, expanded the study of Ramsey's Theorem to infinite cardinals. Let a set $\Omega$ have cardinality $\alpha$. Let the pairs $\{x, y\} \subset$ $\Omega$ be split into a finite number of classes. What is the largest $\beta$ such that there exists a set $S \subset \Omega$ of cardinality $\beta$ which is monochromatic? There are many surprises. For example, assuming the Axiom of Choice, when $\alpha$ is the continuum there is a two-coloring of the pairs so that no monochromatic set $S$ has more than countable size. Erdós also explored Ramsey's Theorem on countable ordinals. Here is a representative beautiful result of Jean Larson: Let the pairs on $\omega^{\omega}$ be colored red and blue. Then there exists either a red triangle or a blue set $S$ of ordinal type $\omega^{\omega}$.

Coloring to avoid a monochromatic set (or proving that this cannot be done) was another problem that fascinated Paul Erdôs over many decades. In 1963 he began [5] the study of perhaps the purest form of the problem. Let $A_{i}, 1 \leq i \leq m$, be $m$ sets in some universe $\Omega$, each with $n$ elements. No assumption about the size of $\Omega$ nor the intersection patterns is made. It is convenient to parametrize $m=k 2^{n-1}$. Erdós showed that if $k<1$, then the family is two-colorable; that is, there exists a two-coloring of the vertices of $\Omega$ such that no set is monochromatic. The proof today: Color Randomly! Let $m(n)$ be the largest value of $m$ such that every family could be two-colored. With this notation $m(n) \geq 2^{n}-1$, Erdős immediately asked for the asymptotics of $m(n)$. In 1964 he showed $m(n) \leq c n^{2} 2^{n}$ by taking random sets in an appropriately chosen $\Omega$. This upper bound 
has not been improved. In 2000 Radhakrishnan and Srinivasan colored an arbitrary family with $k=c \sqrt{n / \ln n}$ by first randomly coloring and then applying an ingenious recoloring algorithm. This result was recently duplicated independently by Cherkashin and by Kozik. Kozik's algorithm is the simplest: Order the vertices randomly. Color a vertex blue unless it would create a blue set; then color it red. Random greedy! Uncle Paul would have been pleased.

Motivated by the difficulty of finding $r(l, k)$, it was natural to extend the class of desired monochromatic objects to include all graphs and not just complete graphs. Thus, in the simplest case, for two given graphs $G$ and $H$, we define the Ramsey number $r(G, H)$ to be the least integer $r$ (guaranteed to exist by Ramsey's Theorem) so that in any red/blue coloring of the edges of the complete graph $K_{r}$, there must always be formed either a red copy of $G$ or a blue copy of $H$. This generalization has proved to be very fruitful, with literally many hundreds of papers dealing with these questions since they were first raised in the early 1970s. (Erdős himself was an author of more than fifty of them!)

One of the earliest (simple) results in this area was the following:

Theorem. $r(G, H) \geq(\chi(G)-1)(c(H)-1)$, where $\chi(G)$ denotes the chromatic number of $G$ and $c(H)$ denotes the cardinality of the largest connected component of $\mathrm{H}$.

An immediate consequence of this is the following elegant result:

Theorem. $r\left(T_{m}, K_{n}\right)=(m-1)(n-1)+1$, where $T_{m}$ denotes a tree on $m$ vertices.

Let us use the usual "arrow" notation $H \rightarrow$ $(G, G)$ to denote the fact that any two-coloring of the vertices of the graph $H$ always produces a monochromatic copy of the graph $G$. Further, denote by $\mathbf{C}(\mathbf{G})$ the class of graphs $H$ for which $H \rightarrow(G, G)$ but such that for any proper subgraph $H^{\prime} \subseteq H$, it is not true that $H^{\prime} \rightarrow(G, G)$. Such graphs $H$ are called Ramsey-minimal for $G$. An interesting unsolved problem is to determine those graphs $G$ for which $\mathbf{C}(\mathbf{G})$ is infinite. It is known that this is the case, for example, when $G$ is 3-connected or $G$ has chromatic number at least 3 or when $G$ is a forest which is not the union of stars. A nice conjecture involving $\mathbf{C}(\mathbf{G})$ is the following: If $\mathbf{C}(\mathbf{G})$ is finite and $G^{\prime}$ is formed from $G$ by adding disjoint edges, then $\mathbf{C}\left(\mathbf{G}^{\prime}\right)$ is also finite.

From the simplest arrow relation $K_{6} \rightarrow\left(K_{3}, K_{3}\right)$ (the celebrated "party" problem), it follows that any graph $H$ containing $K_{6}$ as a subgraph also satisfies $H \rightarrow\left(K_{3}, K_{3}\right)$. Erdős and Hajnal already asked in the 1960s for an example of the graph $H$ not containing
$K_{6}$ as a subgraph for which $H \rightarrow\left(K_{3}, K_{3}\right)$. It was shown that the smallest such graph has eight vertices and is formed by removing a 5-cycle from $K_{8}$. A much more challenging question was to find such a graph which had no $K_{4}$ as a subgraph. This was finally settled by a brilliant construction of Jon Folkman. Unfortunately, his example had more than $10^{10^{10}}$ vertices. This prompted Erdôs to offer a prize for the first example of a "Folkman" graph with fewer than $10^{10}$ vertices, a prize that one of the authors (JS) was proud to claim. Unfazed, the other author (RG) then offered a reward of $\$ 100$ to show that there was a Folkman graph with fewer than $10^{6}$ vertices. This remained unresolved until 2007 when L. Lu constructed a Folkman graph with only 9,697 vertices. The next year this bound was lowered to 941 by Dudek and Rödl. Both of these results used techniques from spectral graph theory. The current record (in 2012) is 786 and is held by Lange, Radziszowski, and Xu. There is some evidence that the best possible bound is below 100 (and RG offers $\$ 100$ for a proof or disproof of this).

Some forty years ago, Erdős and Graham conjectured that in some sense, the complete graph had the largest Ramsey number among all graphs with the same number of edges. Since it is known that $r\left(K_{n}\right)>2^{\frac{n}{2}}$ and $K_{n}$ has $\left(\begin{array}{l}n \\ 2\end{array}\right)$ edges, it was then conjectured that there is an absolute constant $c$ such that for any graph $G$ with $m$ edges, $r(G)<2^{c \sqrt{m}}$. This was finally proven [13] in 2011 by Sudakov. However, the (somewhat) related conjecture that for any graph $H$ with chromatic number $n, r(H) \geq r\left(K_{n}\right)$ is still unresolved.

We say that a graph $H$ is d-degenerate if every subgraph has a vertex of degree at most $d$. Equivalently, there is an ordering of the vertices of $H$ such that each vertex has at most $d$ edges "to the left." A conjecture of Erdős and Stephan Burr from the 1970s has been quite influential:

Burr-Erdós Conjecture. For each positive integer $d$, there is a constant $c(d)$ such that every $d$-degenerate graph $H$ satisfies $r(H)<c(d) n$.

In other words, $d$-degenerate graphs (fixing $d$ ) have linearly growing Ramsey numbers. While this conjecture is still unsettled, it has served as a focal point for a variety of results related to it. The best result in this direction up to now is the recent striking result of Fox and Sudakov which shows that there is a constant $c(d)$ so that any

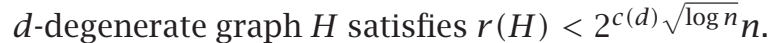
(Close, but no cigar!)

In fact, there are quite a few of the very first questions raised by Erdős which are still unanswered. One of the nicest is the following. Denote by $r(G ; k)$ the least integer $r$ such that if the edges 
of $K_{r}$ are $k$-colored, then a monochromatic copy of $G$ will always be formed. (Again, the existence of $r(G ; k)$ follows from Ramsey's Theorem.) What is the true order of growth for $r\left(C_{3} ; k\right)$ as $k$ tends to infinity (where $C_{t}$ denotes a cycle with $t$ edges)? The best current bounds available are essentially $2^{k}<r\left(C_{3} ; k\right)<2(k+2)$ !. Is it true that for some constant $A$, we have $r\left(C_{3} ; k\right)<A^{k}$ ? Is it true that $r\left(C_{5} ; k\right)>r\left(C_{3} ; k\right)$ ? Can one show that $r\left(C_{2 m+1} ; k\right)>r\left(C_{3} ; k\right)$ for fixed $m$ and $k$ large? Our lack of knowledge here is painfully obvious.

Another direction in Ramsey Theory pioneered by Erdős and the authors (together with P. Montgomery, B. Rothschild, and E. Straus) in a series of papers [6] published some forty years ago had a geometrical flavor. Let's call a (finite) point configuration $C \subseteq \mathbf{E}^{m}$ Ramsey if for every positive integer $r$ there is a number $N=N(r)$ such that for any $r$-coloring of the points in $\mathbf{E}^{N}$, there is always formed a homothetic copy of $C$ which is monochromatic (i.e., a set $C^{\prime}$ obtained by some Euclidean motion of $C$ ). It is known that the Cartesian product of Ramsey sets is Ramsey. Since any 2-point set is Ramsey, then any subset of the vertices of a rectangular parallelepiped is Ramsey. On the other hand, it can be shown that any Ramsey configuration must lie on the surface of some Euclidean sphere. Thus, the collinear set $T=\{0,1,2\}$ of the three vertices of the degenerate triangle $T$ is not Ramsey. In fact, it is a nice exercise to show if the points $\bar{x} \in \mathrm{E}^{n}$ are four-colored by color $(\bar{x})=\left\lfloor\|\bar{x}\|^{2}\right\rfloor(\bmod 4)$, then there is no monochromatic copy of $T$. It is not known if this is possible using only three colors. A long-standing conjecture is the following.

Conjecture A. A configuration is Ramsey if and only if it is spherical.

Recently, an alternative conjecture has been proposed by Leader, Russell, and Walters. Let us call a configuration $C$ transitive if it has a transitive symmetry group. Furthermore, let us say that a configuration $C^{\prime}$ is subtransitive if it is a subset of a transitive configuration. Leader et al. noted that all configurations which had been shown to be Ramsey are in fact subtransitive. Consequently, they conjectured:

Conjecture B. A configuration is Ramsey if and only if it is subtransitive.

Interestingly, neither of these conjectures implies the other. Conjecture A implies that all finite subsets of a circle are Ramsey. On the other hand, it has been shown that almost all 4-point subsets of a circle are not subtransitive. The strongest results on this problem so far are due to Kříž, who showed that $C$ is Ramsey if it has a solvable

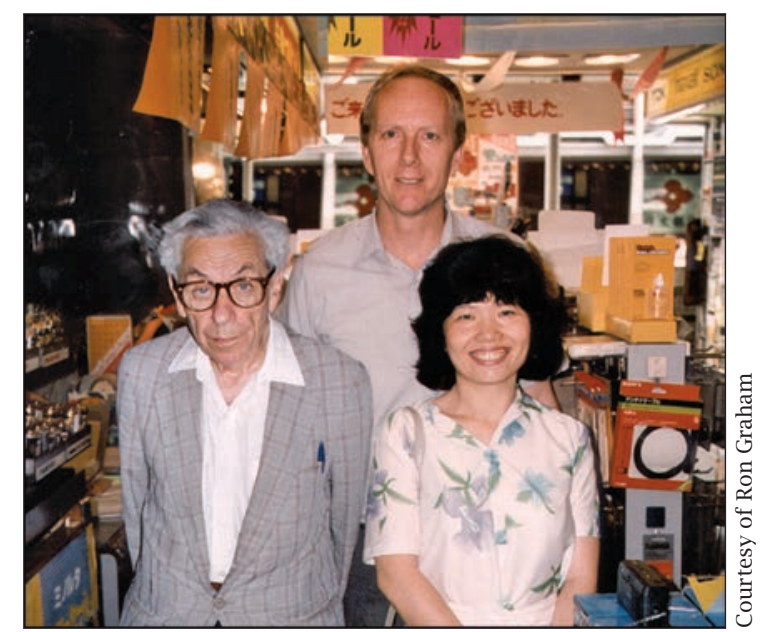

(From I to r) Paul Erdős, Ron Graham, and Fan Chung in Hakone, Japan, in 1986.

transitive group of symmetries. Thus, the set of vertices of a regular $n$-gon is Ramsey.

We close with several tantalizing (simple!) Euclidean Ramsey conjectures.

Conjecture. In any 2-coloring of the points in the plane, every 3-point configuration must occur monochromatically, with the possible exception of the set of three vertices of some fixed equilateral triangle.

It is easy to two-color $\mathbf{E}^{2}$ by half-open alternating red/blue strips of width $\frac{\sqrt{3}}{2}$ which avoids monochromatic copies of the three vertices of a unit equilateral triangle.

On the other hand, we have:

Conjecture. For any 3-point configuration $C_{3}$ of the plane, there is some three-coloring of the points of the plane which contains no monochromatic copy of $C_{3}$.

Clearly, there is a lot more to be done before we have a complete understanding of these questions. Now that Uncle Paul can read proofs from THE BOOK, we are sure that he is annoyed for not having seen the answers while he was still with us!

\section{References}

As general references we give our own books [1], [2], [9]. From the more than 1, 500 papers of Paul Erdős we select a handful that we feel are particularly noteworthy:

\section{References}

[1] N. Alon and J. Spencer, The Probabilistic Method, Wiley, 1992.

[2] F. Chung and R. L. GRAham, Erdös on Graphs, His Legacy of Unsolved Problems, A K Peters, 1998.

[3] P. ERDŐs, Some remarks on the theory of graphs, Bull. Amer. Math. Soc. 53 (1947), 292-294. 
[4] _ Graph theory and probability II, Canad. J. Math. 13 (1961), 346-352.

[5] _ On a combinatorial problem I, Nordisk. Mat. Tidskr. 11 (1963), 5-10.

[6] P. ERDŐS, R. L. GRAHAM, P. MONTGOMERY, B. L. ROTHSCHILD, J. H. SPENCER, and E. G. STRAUS, Euclidean Ramsey theorems, I., J. Combinatorial Theory Ser. A 14 (1973), 341-363.

[7] P. ERDős and R. RADO, A combinatorial theorem, $J$. London Math. Soc. 25 (1950), 249-255.

[8] A A partition calculus in set theory, Bull. Amer. Math. Soc. 62 (1956), 427-489.

[9] R. L. Graham, B. L. Rothschild, and J. SPencer, Ramsey Theory, Wiley, 1980.

[10] P. ERDŐS, S. SUEN, and P. WINKLER, On the size of a random maximal graph, Random Structures \& Algorithms 6 (1995), 309-315.

[11] P. ERDŐS and G. SZEKERES, A combinatorial problem in geometry, Compositio Math 2 (1935), 463-470.

[12] J. H. KIM, The Ramsey number $R(3, t)$ has order of magnitude $t^{2} / \ln t$, Random Structures \& Algorithms 7 (1995), 173-207.

[13] B. SUDAKOV, A conjecture of Erdős on graph Ramsey numbers, Advances in Mathematics 227 (2011), 601609.

\section{Jean-Pierre Kahane}

\section{Bernoulli Convolutions and Self-similar Measures after Erdős}

\section{A Personal hors d'øeuvre}

I never collaborated with Paul Erdős and didn't meet him very frequently. However, I regarded him, and believe he regarded me, as a friend. Several hundreds of mathematicians all around the world may feel the same-anyway it is a strong personal feeling of mine. I wasn't his closest friend by far. His closest French friend was Jean-Louis Nicolas, who is much younger and entertained Paul in Limoges and Lyons. Jean-Louis collaborated with Paul and wrote beautiful papers about him [N1], [N2], [N3]. As a detail let me mention their common interest in highly composite numbers, a term coined by Ramanujan for numbers like 60 or 5040 that have more divisors than any smaller number [E1], [EN], [N4]; my own interest was in the implicit occurrence of the notion in Plato's utopia: 5040 is the best number of citizens in a city because it is highly divisible (Laws, 771c). Through Nicolas my Erdős number is 2.

I met Paul Erdős for the first time in Amsterdam in 1954 at the International Congress of Mathematicians. He was a famous mathematician, a famous nonwinner of the Fields Medal in 1950. He was not a member of the Hungarian delegation; he came from Jerusalem, where he had already worked with Aryeh Dvoretsky. I had just published

Jean-Pierre Kahane is professor emeritus at the Université Paris-Sud à Orsay. His email address is jeanpierre.kahane@u-psud.fr. my doctoral thesis, written under the supervision of Szolem Mandelbrojt, and it had very little to do with the mathematics of Erdős. Our common point was that my wife, who is of Hungarian origin, was there and spoke Hungarian. Since then he always asked me about her and her health and later about my children and grandchildren-epsilons and epsilon-squares-and he asked me about politics and about mathematical questions he had in mind: regularly the last few times about an exponent which describes how close to a constant the absolute value of a polynomial of degree $n$ can be when the coefficients have absolute value 1 . He remembered the exponent I had published and regularly forgot.

Shortly after 1954, I read the book of Paul Lévy on Brownian motion. Paul Lévy was impressed by a discovery of Dvoretsky, Erdős, and Kakutani on multiple points of the Brownian motion in the plane, namely, the existence of multiple points of nondenumerable order [DEK1], [DEK2]. It is a beautiful result indeed. Here is a reinforced version of the theorem, due to Jean-François Le Gall: Consider a compact set on the real line with empty interior, $K$, and a plane Brownian motion; then the plane Brownian motion has almost surely a $K$-multiple point $z$, meaning that the reciprocal image of $z$ is homeomorphic to $K$ through an increasing mapping [LG]. In turn this result impressed Wendelin Werner when he began to work on the plane Brownian motion with Le Gall as advisor. In this way Erdős (1954) is related to the Fields Medal received by Werner in 2006.

\section{The Main Course: Bernoulli Convolutions}

In 2000 Yuval Peres, William Schlag, and Boris Solomyak published a beautiful study on "Sixty years of Bernoulli convolutions" [PSS]. The starting point was the seminal papers of Erdős in 1939 and 1940. Since 2000 and quite recently, Bernoulli convolutions and self-similar measures were the subjects of bright contributions, including the brilliant lecture of Elon Lindenstrauss at the Erdôs Centennial July 2013 [L]. Old conjectures remain and new ones appear. The quite recent paper of Pablo Shmerkin [Sh] gives new results and an excellent exposition of the whole subject. I shall try to describe the impulse given by Erdôs and how things progressed with the notations in use today.

Given $\lambda$ in the open interval $(0,1)$, we consider the random series

$$
\sum_{n=0}^{\infty} \pm \lambda^{n}
$$

where the signs + and - are chosen at random, independently each from the others, with probability $1 / 2$. The sum is a random variable whose 
distribution is the Bernoulli convolution under consideration,

$$
v_{\lambda}=\underset{n=0}{\stackrel{\infty}{*}} \frac{1}{2}\left(\delta_{\lambda^{n}}+\delta_{-\lambda^{n}}\right),
$$

and the characteristic function is the infinite product

$$
\hat{v}_{\lambda}(\xi)=\prod_{n=0}^{\infty} \cos \lambda^{n} \xi .
$$

When $\lambda=\frac{1}{2}, v_{\lambda}$ is nothing but $\frac{1}{4} \times$ Lebesgue measure on $(-2,2)$. When $\lambda<\frac{1}{2}$, $v_{\lambda}$ is carried by a Cantor set whose ratio of dissection is $\lambda$. This set has Hausdorff dimension $\frac{\log 2}{\log 1 / \lambda}$; it is self-similar, meaning that it is the union of two disjoint homothetic copies with ratio $\lambda$; and $v_{\lambda}$ is the natural measure thereon, giving equal masses to equal portions. When $\lambda>\frac{1}{2}$, (1) takes every value between $\frac{-1}{1-\lambda}$ and $\frac{1}{1-\lambda}$ and $\nu_{\lambda}$ is a self-similar measure, meaning the average of two copies obtained by similarities of ratio $\lambda$ (affine functions with main coefficient $\lambda$ ). It has been known since $1935[\mathrm{JW}],[\mathrm{KW}]$ that $v_{\lambda}$ is either absolutely continuous or purely singular with respect to the Lebesgue measure

(4) $v_{\lambda} \ll$ Lebesgue or $v_{\lambda} \perp$ Lebesgue.

To decide between these two possibilities according to the value of $\lambda$ is the first and the main question when $\lambda>\frac{1}{2}$.

This question was considered by Erdôs in two papers published in 1939 and 1940 by the American Journal of Mathematics [E2], [E3]. The first deals with the question: when is

$$
\limsup _{\xi \rightarrow \infty} \hat{v}_{\lambda}(\xi)>0 ?
$$

According to Riemann-Lebesgue, this can't happen when $v_{\lambda}$ is absolutely continuous; therefore (5) implies that $v_{\lambda}$ is singular.

The question makes sense for $\lambda>\frac{1}{2}$ as well as $\lambda<\frac{1}{2}$, but the implications are quite different: when $\lambda<\frac{1}{2}, v_{\lambda}$ is singular and (5) has a meaning in the Riemann-Cantor theory of trigonometric series [Z] ; when $\lambda>\frac{1}{2}$ it is a way-the only way we have up to now-to find $\lambda$ for which $v_{\lambda} \perp$ Lebesgue. In any case it is convenient to introduce

$$
\theta=1 / \lambda
$$

and to use the formula

$$
\hat{v}_{\lambda}\left(t \theta^{N} \pi\right)=\prod_{n=1}^{N} \cos \left(t \theta^{n} \pi\right) \prod_{n=0}^{\infty} \cos \left(t \lambda^{n} \pi\right)
$$

where $1 \leq t<\theta$. When $\theta$ is an integer $>2$, choosing $t=1$ gives (5). The same works whenever all $\theta^{n}$ are very close to integers. Erdôs observed that it is the case indeed when $\theta$ is a Pisot-Vijayaraghavan or Pisot number, that is, an algebraic integer whose conjugates (other than $\theta$ itself) lie inside the unit circle of the complex plane (Erdós' condition).

It was the beginning of a long story where Raphaël Salem played the major role. First, including the integers larger than 2 among the Pisot numbers, the Erdoss condition is necessary and sufficient for (5) to hold. Then, considering the case $\lambda<\frac{1}{2}$, the Erdós condition is necessary and sufficient for the support of $v_{\lambda}$, the Cantor set described by (1), to be a set of uniqueness, or $U$-set, for trigonometric series; that is, the only trigonometric series that converges to 0 out of the set is the null series. This is one of the most striking relations between the theory of numbers and trigonometric series [S]. Finally, the set of Pisot numbers is closed [S]. Already $\frac{1+\sqrt{5}}{2}$ and the real root of $x^{3}-x-1=0$ had been recognized as Pisot numbers lying in the interval $(1,2)$ : it happens that the first is the smallest accumulation point of Pisot numbers, and the second, the smallest Pisot number [BDGPS].

The set of Pisot numbers, denoted by $S$, is now well understood [BDGPS], [M]. It is not the case for a companion introduced by Salem, the set $T$ of algebraic integers $\tau$ whose conjugates other than $\tau$ lie in the closed disc $|z| \leq 1$ with one at least (then all but two) on the boundary $|z|=1$. Those numbers $\tau$ are now called Salem numbers. Every $\theta \in S$ is a limit in both directions of a sequence of $\tau \in T$, and no other accumulation point of $T$ is known [S]. Is 1 an accumulation point for T? A section of [PSS], entitled "Bernoulli convolutions and Salem numbers," is devoted to this question, still unsolved.

The second paper of Erdős [E3] goes in the opposite direction: when is $v_{\lambda} \ll$ Lebesgue? His approach is another question: when is

$$
\hat{v}_{\lambda}(\xi)=O\left(\xi^{-\alpha}\right) \quad(\xi \rightarrow \infty)
$$

for some $\alpha>0$ ? His answer is that (8) holds for some $\alpha \simeq \alpha(\lambda)>0$ for almost all $\lambda$. More exactly, given $\alpha>0$, there exists $\delta>0$ such that (8) holds for almost all $\lambda \in(1-\delta, 1)$. When (8) holds, we can make use of the identity

$$
\hat{v}_{\sqrt{\lambda}}(\xi)=\hat{v}_{\lambda}(\xi) \hat{v}_{\lambda}(\xi \sqrt{\lambda})
$$

and its analogues to get

(10)

$\hat{v}_{\sqrt{\lambda}}(\xi)=O\left(\xi^{-2 \alpha}\right), \hat{v}_{\lambda^{1 / k}}(\xi)=O\left(\xi^{-k \alpha}\right)(\xi \rightarrow \infty)$.

As soon as $k \alpha>1 / 2$, the measure $v_{\lambda^{1 / k}}$ is absolutely continuous with density in $L^{2}$; when $k \alpha>1$ the density is continuous, and so on. That is a way to obtain information for $\lambda$ near 1 from information for $\lambda$ belonging to any interval. Anyway, $v_{\lambda} \ll$ Lebesgue for almost all $\lambda$ belonging to some interval $(1-\delta, 1)$.

No progress was made for a long time. Adriano Garcia in 1962 gave a new method to find explicit 
values of $\lambda$ for which $\nu_{\lambda} \ll$ Lebesgue, with bounded density. Here is a typical result of his: If $\theta$ is an algebraic integer with norm 2 and all conjugates outside the disc $|z| \leq 1$, then $v_{\lambda} \ll$ Lebesgue with bounded density [G].

The short report that I published on these questions in 1971 contains an exposition of Erdős's proof in twelve lines (tersely written, according to [PSS]) with an improved conclusion: $E_{0}$, the $\lambda$-set such that (8) fails for every $\alpha>0$, is 0-dimensional. In the statements of Erdős that I gave above, "almost all" can be replaced by "except on a 0 -dimensional set" [K]. The combinatorial argument was later developed by Peres, Schlag, and Solomyak ([PSS], Proposition 6.1) and used by Shmerkin ([Sh], Propositions 2.2 and 2.3). However, the numerical consequence expressed in [PSS] is rather poor: if we want to have (8) with $\alpha=0.6$ (therefore $v_{\lambda}$ is absolutely continuous with density in $L^{2}$ ) when $\lambda \in(1-\delta, 1) \backslash G$ with $\operatorname{dim} G<1$, the combinatorial argument provides $1-\delta=2^{-2^{-10}}$, that is, $\delta \simeq 0.00066$.

The first breakthrough was realized by Solomyak in 1995: $v_{\lambda}$ is absolutely continuous with density in $L^{2}$ for almost all $\lambda$ in $\left(\frac{1}{2}, 1\right)$ [So], [PS]. The second is quite recent: the exceptional set for which $v_{\lambda}$ is singular is not only of Lebesgue measure 0 , but it is also 0-dimensional [Sh]. This last result, due to Pablo Shmerkin, echoes and uses another quite recent and deep discovery of Michael Hochman: Except for a $\lambda$-set of dimension $0, v_{\lambda}$ has dimension 1 , meaning that $v_{\lambda}(B)=0$ when $\operatorname{dim} B<1$ (we assume always $\frac{1}{2}<\lambda<1$ ) [H].

Extensions and new developments on nonsymmetric Bernoulli convolutions (+ and - in (1) having unequal probabilities) and on self-similar measures (not restricted to the real line) can be found in [PSS], [L], [Sh] and the references therein.

Going back to the questions: 1 ) when is $v_{\lambda} \ll$ Lebesgue? 2) when is (8) valid for some $\alpha>0$ (power decay of $\hat{v}_{\lambda}$ )? the answer is that they hold whenever $\lambda \in\left(\frac{1}{2}, 1\right) \backslash E, E$ being a 0 -dimensional set. $E$ is not the same in question 1 and question 2; let us write $E_{1}$ and $E_{2}$ accordingly. We know that $E_{1}$ contains the inverses of the Pisot numbers $<2$. It is easy to see that $E_{2}$ contains the inverses of the Salem as well as the Pisot numbers, and also $\theta^{-1}$ as soon as $\theta^{n}$ tends to 0 modulo $1(n \rightarrow \infty)$. The study of those $\theta$ by Charles Pisot in 1938 should be mentioned here [P]. First, the result the set of those $\theta$ is denumerable. Then, the question does it contain anything other than Pisot numbers? And finally the proof: it is easy, but it is a paradigm for the combinatorial argument now known as Erdős-Kahane [PSS], [Sh].

\section{Dreams and Goals}

All investigations on the asymptotic behavior of the infinite product (3) rely on the formula (7):

$$
\hat{v}_{\lambda}\left(t \theta^{N} \pi\right)=\prod_{n=1}^{N} \cos \left(t \theta^{n} \pi\right) \prod_{n=0}^{\infty} \cos \left(t \lambda^{n} \pi\right),
$$

where $\theta=1 / \lambda$ and $1 \leq t<\theta$. The second product is bounded, and the first involves the behavior of $t \theta^{n}$ modulo 1. Erdós in 1939 used the fact that $\theta^{n}$ modulo 1 tends to 0 rapidly when $\theta$ is a Pisot number. Let us look at the most classical result, going back to Hardy and Littlewood [HL] and to Hermann Weyl [W]: given $t$, the distribution of $t \theta^{n}$ modulo 1 is uniform on $\mathbb{R} / \mathbb{Z}$ for almost every $\theta$. It implies that

$$
\begin{aligned}
& \lim _{N \rightarrow \infty} \frac{1}{N} \sum_{n=1}^{N} \log \left|\cos \left(t \theta^{n} \pi\right)\right| \\
& \quad=\int_{0}^{1} \log |\cos \pi x| d x=-\log 2^{1} ;
\end{aligned}
$$

therefore, given $t$, almost every $\lambda$ in $(0,1)$ satisfies

$$
\hat{v}_{\lambda}\left(t \theta^{N} \pi\right)=O\left(2^{-N+\varepsilon N}\right) \quad(N \rightarrow \infty)
$$

and

$$
\hat{v}_{\lambda}\left(t \theta^{N} \pi\right)=\Omega\left(2^{-N-\varepsilon N}\right) \quad(N \rightarrow \infty)
$$

for all $\varepsilon>0$. For almost every $\lambda$ in $(0,1)(11)$ and (12) hold a.e. in $t$, but $O(\cdot)$ and $\Omega(\cdot)$ depend on $\lambda$ and $t$. Let us write

(13) $g(\lambda)=\sup \left\{\alpha: \hat{v}_{\lambda}(\xi)=O\left(\xi^{-\alpha}\right)(\xi \rightarrow \infty)\right\}$.

According to (12) we have a.e. in $\lambda$,

$$
g(\lambda) \leq-\frac{\log 2}{\log \lambda} .
$$

If we forget that $O(\cdot)$ in (11) depends on $\lambda$ and $t$, we may dream and ask the question: Is it true that

$$
g(\lambda)=-\frac{\log 2}{\log \lambda}
$$

for almost every $\lambda$ in $(0,1)$ ? That is asked with a wrong factor $\frac{1}{2}$ in [K]; then a negative answer was given by Peres and Solomyak [PSo]. Here is what they proved. Writing

$$
\lambda_{n}=\left(\begin{array}{c}
2 n \\
n
\end{array}\right) 2^{-2 n}
$$

$\left(\lambda_{1}=\frac{1}{2}, \lambda_{2}=\frac{3}{8}, \lambda_{3}=\frac{5}{16}, \ldots, \lambda_{n} \sim \frac{1}{\sqrt{\pi n}}(n \rightarrow \infty)\right)$, we have

$$
\begin{cases}\hat{v}_{\lambda} \in L^{2 n}(\mathbb{R}) & \text { for almost all } \lambda \in\left(\lambda_{n}, 1\right), \\ \hat{v}_{\lambda} \notin L^{2 n}(\mathbb{R}) & \text { for all } \lambda<\lambda_{n}\end{cases}
$$

We recover Solomyak's theorem when $n=1$. The negative answer was obtained for $n=2$ and $\lambda=\frac{1}{4}$.

${ }^{1} 20$ denoting the mean value $2 n(\log |\cos \pi x|)=2 n$

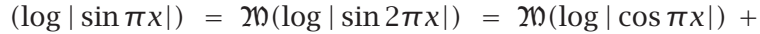
$20(\log |\sin \pi x|)+\log 2$. I am indebted to Mrs Anne Raoult for this footnote. 
A weak point of the beautiful result (17) is that all $\lambda_{n}$ lie in $\left(0, \frac{1}{2}\right)$. In order to have estimates on $\left(\frac{1}{2}, 1\right)$, Peres and Schlag considered weighted $L^{2}$ instead of $L^{2 n}$. Here is a typical result: Suppose $J=\left[\lambda_{0}, \lambda_{0}^{\prime}\right] \subset\left[\frac{1}{2}, 0.68\right]$ and $\lambda_{0}^{1+2 \gamma}>\frac{1}{2}$; then

$$
\iint_{\mathbb{R} \times J}\left|\hat{v}_{\lambda}(\xi)\right|^{2}|\xi|^{2 \gamma} d \xi d \lambda<\infty ;
$$

hence

$$
\begin{aligned}
& \hat{v}_{\lambda}(\xi)=o\left(\xi^{-\gamma}\right)(\xi \rightarrow \infty) \\
& \text { for a.e. } \lambda \in J \text { [PSS, section 7]. }
\end{aligned}
$$

$[\mathrm{G}]$

$[\mathrm{H}]$

H] Michael Hochman, On self-similar sets with overlap and inverse theorems for entropy, Ann. of Math. (2) 180 (2014), 773-822.

[HL] G. H. HaRdy and J. E. LitTlewOOD, Some problems of diophantine approximation, Acta Math. 37 (1914), 155-191; cf. p. 183.

[JW] Børge JESSEN and AUREL WintNer, Distribution functions and the Riemann zeta function,Trans. Amer. Math. Soc. 38 (1935), 48-88.

[K] JeAn-Pierre Kahane, Sur la distribution de certaines séries aléatoires, Colloque Th. Nombres [1969, Bordeaux], Bull. Soc. Math. France, Mémoires 25 (1971), 119-122.

This is far from (15), but it is an important step in estimating $g(\lambda)$.

A general goal is the study of $g(\lambda)$ on $(0,1)$. What happens "in general"? What happens in exceptional cases, or particular cases, has interesting relations with the theory of numbers. We already saw that $g(\lambda)=0$ for Pisot and Salem numbers. When $\lambda$ is a Garcia number, it was proved recently that $g(\lambda)>0[\mathrm{DFW}]$.

Let me express ancient problems $[\mathrm{S}]$ as dreams: 1) " $\theta^{n}$ modulo 1 tends to 0 " implies that $\theta$ is Pisot.

2) A limit of Salem numbers is Pisot.

And here is the main dream on Bernoulli convolutions:

$v_{\lambda} \perp$ Lebesgue implies that $\lambda$ is Pisot.

The behavior of powers modulo 1 is the matter of new investigations and problems [B, BM, K1].

\section{References}

[B] Yann Bugeaud, Distribution modulo 1 and Diophantine Approximation, Cambridge Tracts in Mathematics 193 (2012).

[BDGPS] MARIE-José BERTIN, ANNETTE DeCOMPSGuilloux, M. Grandet-Hugot, Martine PathiauX-Delefosse and JeAn-Pierre SCHreIBER, Pisot and Salem Numbers, Birkhäuser, 1992.

[BM] Yann Bugeaud and Nikolay Moshchevitin, On fractional parts of powers of real numbers close to 1, Math. Z. , to appear.

[DEK1] ARYEH DVORETSKY, PAUl ERdős and SHIZUO KAKUTANI, Multiple points of paths of Brownian motion in the plane, Bull. Res. Council Israël F3 (1954), 364-371.

[DEK2] _ Points of multiplicity $c$ of plane Brownian paths, Bull. Res. Council Israël F7 (1958), 175-180.

[DFW] XING-Rong DAI, De-Jun Feng, and YANG WANG, Refinable functions with noninteger dilations, $J$. Funct. Anal. 250 (2007), 1-20.

[E1] PAUL ERDŐs, On highly composite numbers, $J$. London Math. Soc. 19 (1944), 130-133.

[E2] _ On a family of symmetric Bernoulli convolutions, Amer. J. Math. 61 (1939), 974-975.

[E3] _ On smoothness properties of Bernoulli convolutions, Amer. J. Math. 62 (1940), 180-186.

[EN] PAUl ERDős and JeAN-LOUIS Nicolas, Répartition des nombres super abondants, Bulletin Soc. Math. France 103 (1975), 65-90.
[K1] JEAN-PIERRE KAHANE, Sur la répartition des puissances modulo 1, C.R. Acad. Sci. Paris, série 1, 352 (2014) 383-385.

[KW] RICHARD KERSHNER and AUREL WINTNER, On symmetric Bernoulli convolutions, Amer. J. Math. 57 (1935), 541-548.

[L] ELON LINDENSTRAUSs, Bernoulli convolutions and self similar measures, Invited Plenary Lecture, Erdős Centennial, Budapest, July 2013.

[LG] JEAN-FrANÇOIS LE GALL, Le comportement du mouvement brownien entre deux instants où il passe par un point double, J. Functional Analysis 71 (1987), 246-262.

[M] YVES MeYER, Algebraic Numbers and Harmonic Analysis, North-Holland, 1972.

[N1] JEAN-Louis Nicolas, Paul Erdös 1913-1996, Universalia 1997, la politique, les connaissances, la culture en 1996, Encyclopedia Universalis Paris, 1997, p. 471.

[N2] , Discours prononcé à l'occasion du doctorat honoris causa de l'Université de Limoges à Monsieur le Professeur Paul Erdős, Gazette des mathématiciens 33 (1987), 81-85.

[N3] , Statistical Properties of Partitions, Paul Erdós and His Mathematics I, Bolyai Math. Studies, 11, Budapest, 2002, pp. 537-547.

[N4]__ On Landau's function $g(n)$, The mathematics of Paul Erdôs. I, R. L. Graham and J. Nešetřil, eds., Algorithms and Combinatorics, 13, Springer, 1997, pp. 228-240; reedited by Springer, 2013, pp. 207-220. nombres algébriques, Ann. Schola. Norm. Sup. Pisa 2 (1938), 205-248.

[PS] YuVAl Peres and Wilhelm Schlag, Smoothness of projections, Bernoulli convolutions, and the dimension of exceptions, Duke Math. J. 102 (2) (2000),193-251.

[PSo] YuVAl Peres and Boris SOlOMYAK, Self-similar measures and intersections of Cantor sets, Trans. Amer. Math. Soc. 350 (2007), 1-20.

[PSS] Yuval Peres, Wilhem Schlag, and Boris SolOMYAK, Sixty Years of Bernoulli Convolutions, Fractal geometry and stochastics, II (Greifswald/Koserow, 1998), vol. 46, Progr. Probab., Birkhäuser, Basel, 2000 pp. 39-65.

[S] RAPHAËL SALEM, Algebraic Numbers and Fourier Analysis, Heath, 1963.
[P] ChARLes Pisot, La répartition modulo 1 et les 
[Sh] PABlo Shmerkin, On the exceptional set for absolute continuity of Bernoulli convolutions, Geom. Funct. Anal. 24 (2014), 946-958.

[So] BORIS SOLOMYAK, On the random series $\sum \pm \lambda^{n}$ (an Erdős problem), Ann. of Math. (2), 142 (3) (1955), 611-625.

[W] HERMANN WEYL, Über die Gleichverteilung von Zahlen mod. Eins, Math. Annalen 77 (1916), 313352, Satz 21.

[Z] Antoni Zygmund, Trigonometric Series. I, II, Cambridge Univ. Press, 1959.

\section{Melvyn B. Nathanson}

\section{Paul Erdős and Additive Number Theory Additive Bases}

Paul Erdős, while he was still in his twenties, wrote a series of extraordinarily beautiful papers in additive and combinatorial number theory. The key concept is additive basis.

Let $A$ be a set of nonnegative integers, let $h$ be a positive integer, and let $h A$ denote the set of integers that can be represented as the sum of exactly $h$ elements of $A$, with repetitions allowed. A central problem in additive number theory is to describe the sumset $h A$. The set $A$ is called an additive basis of order $h$ if every nonnegative integer can be represented as the sum of exactly $h$ elements of $A$. For example, the set of squares is a basis of order 4 (Lagrange's theorem), and the set of nonnegative cubes is a basis of order 9 (Wieferich's theorem).

The set $A$ of nonnegative integers is an asymptotic basis of order $h$ if $h A$ contains every sufficiently large integer. For example, the set of squares is an asymptotic basis of order 4 but not of order 3 . The set of nonnegative cubes is an asymptotic basis of order at most 7 (Linnik's theorem) and, by considering congruences modulo 9 , an asymptotic basis of order at least 4 . The Goldbach conjecture implies that the set of primes is an asymptotic basis of order 3. Helfgott [18] recently completed the proof of the ternary Goldbach conjecture: Every odd integer $n \geq 7$ is the sum of three primes.

The modern theory of additive number theory began with the work of Lev Genrikhovich Shnirel'man (1905-1938). In an extraordinary paper [38] published in Russian in 1930 and republished in an expanded form [39] in German in 1933, he proved that every sufficiently large integer is the sum of a bounded number of primes. Not only did Shnirel'man apply the Brun sieve, which Erdôs subsequently developed into one of the most powerful tools in number theory, but he also introduced a

Melvyn B. Nathanson is professor of mathematics at Lehman College (CUNY). His email address is melvyn. nathanson@1ehman. cuny.edu. new density for a set of integers that is exactly the right density for the investigation of additive bases. (For a survey of the classical bases in additive number theory, see Nathanson [28].)

\section{Shnirel'man Density and Essential Components}

The counting function $A(x)$ of a set $A$ of nonnegative integers counts the number of positive integers in $A$ that do not exceed $x$, that is,

$$
A(x)=\sum_{\substack{a \in A \\ 1 \leq a \leq x}} 1 .
$$

The Shnirel'man density of $A$ is

$$
\sigma(A)=\inf _{n=1,2, \ldots} \frac{A(n)}{n} .
$$

The sum of the sets $A$ and $B$ is the set $A+B=$ $\{a+b: a \in A$ and $b \in B\}$. Shnirel'man proved the fundamental sumset inequality:

$$
\sigma(A+B) \geq \sigma(A)+\sigma(B)-\sigma(A) \sigma(B) .
$$

This implies that if $\sigma(A)>0$, then $A$ is a basis of order $h$ for some $h$. This does not apply directly to the sets of $k$ th powers and the set of primes, which have Shnirel'man density 0 . However, it is straightforward that if $\sigma(A)=0$ but $\sigma\left(h^{\prime} A\right)>0$ for some $h^{\prime}$, then $A$ is a basis of order $h$ for some $h$.

Landau conjectured the following strengthening of Shnirel'man's addition theorem, which was proved by Mann [23] in 1942:

$$
\sigma(A+B) \geq \min (1, \sigma(A)+\sigma(B)) .
$$

Artin and Scherk [1] published a variant of Mann's proof, and Dyson [4], while an undergraduate at Cambridge, generalized Mann's inequality to $h$-fold sums. Nathanson [27] and Hegedüs, Piroska, and Ruzsa [17] have constructed examples to show that the Shnirel'man density theorems of Mann and Dyson are best possible.

We define the lower asymptotic density of a set $A$ of nonnegative integers as follows:

$$
d_{L}(A)=\liminf _{n=1,2, \ldots} \frac{A(n)}{n} .
$$

This is a more natural density than Shnirel'man density. A set $A$ with asymptotic density $d_{L}(A)=0$ has Shnirel'man density $\sigma(A)=0$, but not conversely. A set $A$ with asymptotic density $d_{L}(A)>0$ is not necessarily an asymptotic basis of finite order, but $A$ is an asymptotic basis if $d_{L}(A)>0$ and $\operatorname{gcd}(A)=1$ (cf. Nash and Nathanson [24]).

The set $B$ of nonnegative integers is called an essential component if

$$
\sigma(A+B)>\sigma(A)
$$

for every set $A$ such that $0<\sigma(A)<1$. Shnirel'man's inequality implies that every set of positive Shnirel'man density is an essential 
component. There exist sparse sets of zero asymptotic density that are not essential components. Khinchin [20] proved that the set of nonnegative squares is an essential component. Note that the set of squares is a basis of order 4. Using an extremely clever elementary argument, Erdős [6], at the age of twenty-two, proved the following considerable improvement: Every additive basis is an essential component. Greatly impressed, Landau celebrated this result in his 1937 Cambridge Tract Über einige neuere Fortschritte der additiven Zahlentheorie [22].

Plünnecke [33], [34], [35] and Ruzsa [37] have made important contributions to the study of essential components.

\section{The Erdós-Turán Conjecture}

In another classic paper, published in 1941, Erdős and P. Turán [5] investigated Sidon sets. The set $A$ of nonnegative integers is a Sidon set if every integer has at most one representation as the sum of two elements of $A$. They concluded their paper as follows:

Let $f(n)$ denote the number of representations of $n$ as $a_{i}+a_{j}, \ldots$. If $f(n)>0$ for $n>n_{0}$, then $\lim \sup f(n)=\infty$. Here we may mention that the corresponding result for $g(n)$, the number of representations of $n$ as $a_{i} a_{j}$, can be proved.

The additive statement is still a mystery. The Erdős-Turán conjecture, that the representation function of an asymptotic basis of order 2 is always unbounded, is a major unsolved problem in additive number theory.

Many years later, in 1964, Erdős [7] published the proof of the multiplicative statement. This proof was later simplified by Nešetřil and Rödl [32] and generalized by Nathanson [26].

Long ago, while a graduate student, I searched for a counterexample to the Erdős-Turán conjecture. Such a counterexample might be extremal in several ways. It might be "thin" in the sense that it contains few elements. Every asymptotic basis of order $h$ has counting function $A(x) \geq c x^{1 / h}$ for some $c>0$ and all sufficiently large $x$. We call an additive basis of order $h$ thin if $A(x) \leq c^{\prime} x^{1 / h}$ for some $c^{\prime}>0$ and all sufficiently large $x$. Thin bases exist. The first examples were constructed in the 1930s by Raikov [36] and by Stöhr [40], and Cassels [2], [29] later produced another important class of examples.

Alternatively, an asymptotic basis $A$ of order $h$ might be extremal in the sense that no proper subset of $A$ is an asymptotic basis of order $h$. This means that removing any element of $A$ destroys every representation of infinitely many integers. It is not obvious that minimal asymptotic bases

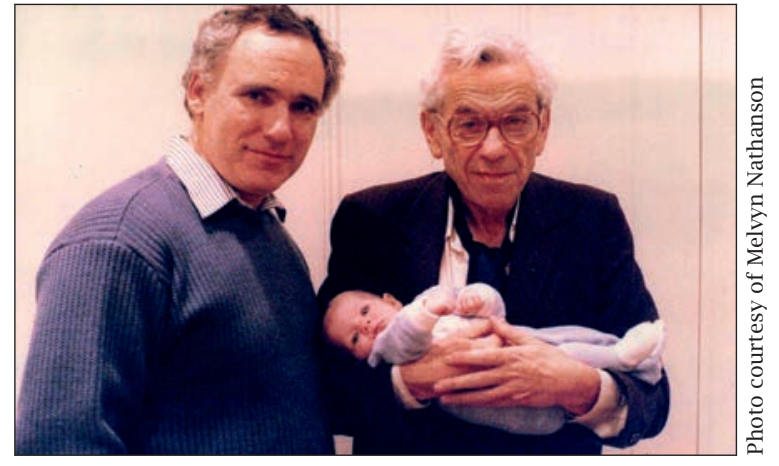

Mel Nathanson with Paul Erdős, who is holding Mel's infant son, Alex, in 1988.

exist, but I was able to construct asymptotic bases of order 2 that were both thin and minimal [25]. Of course, none was a counterexample to the Erdős-Turán conjecture.

Stöhr [41] gave the first definition of minimal asymptotic basis, and Härtter [16] gave a nonconstructive proof that there exist uncountably many minimal asymptotic bases of order $h$ for every $h \geq 2$.

There is a natural dual to the concept of a minimal asymptotic basis. We call a set $A$ an asymptotic nonbasis of order $h$ if is not an asymptotic basis of order $h$, that is, if there are infinitely many positive integers not contained in the sumset $h A$. An asymptotic nonbasis of order $h$ is maximal if $A \cup\{b\}$ is an asymptotic basis of order $h$ for every nonnegative integer $b \notin A$. The set of even nonnegative integers is a trivial example of a maximal nonbasis of order $h$ for every $h \geq 2$, and one can construct many other examples that are unions of the nonnegative parts of congruence classes. It is difficult to construct nontrivial examples.

I discussed this and other open problems in my first paper [25] in additive number theory. I did not know Erdős at the time, but I mailed him a preprint of the article. It still amazes me that he actually read this paper sent to him out of the blue by a completely obscure student, and he answered with a long letter in which he discussed his ideas about one of the problems. This led to correspondence, meetings, and joint work over several decades.

\section{Extremal Properties of Bases}

Here is a small sample of results on minimal bases and maximal nonbases.

Nathanson and Sarközy [31] proved that if $A$ is a minimal asymptotic basis of order $h \geq 2$, then $d_{L}(A) \leq 1 / h$. The proof uses Kneser's theorem [21] on the asymptotic density of sumsets, one of the most beautiful and most forgotten theorems in additive number theory. A well-known special case 
is Kneser's theorem for the sum of finite subsets of a finite abelian group.

Erdôs and Nathanson [14] proved that for every $h \geq 2$, there exist minimal asymptotic bases of order $h$ with asymptotic density $1 / h$. Moreover, for every $\alpha \in(0,1 /(2 h-2))$, there exist minimal asymptotic bases of order $h$ with asymptotic density $\alpha$. In particular, for every $\alpha \in(0,1 / 2]$ there exist minimal asymptotic bases of order 2 with asymptotic density $\alpha$.

Does every asymptotic basis $A$ of order 2 contain a minimal asymptotic basis of order 2 ? Sometimes. Let $f(n)$ count the number of representations of $n$ as the sum of two elements of $A$. If $f(n)>c \log n$ for some $c>(\log (4 / 3))^{-1}$ and all sufficiently large $n$, then $A$ contains a minimal asymptotic basis of order 2 (Erdős-Nathanson [13]). This result is almost certainly not best possible.

Does every asymptotic basis of order 2 contain a minimal asymptotic basis of order 2? No. There exists an asymptotic basis $A$ of order 2 with the following property: If $S \subseteq A$, then $A \backslash S$ is an asymptotic basis of order 2 if and only if $S$ is finite (Erdős-Nathanson [12]).

There exist "trivial" maximal asymptotic nonbases of order $h$ consisting of unions of arithmetic progressions [25]. However, for every $h \geq 2$ there also exist nontrivial maximal asymptotic nonbases of order $h$ (Erdős-Nathanson [8], [11] and Deshouillers and Grekos [3]).

Is every asymptotic nonbasis of order $h$ a subset of a maximal asymptotic nonbasis of order $h$ ? Sometimes. If $A \cup S$ is an asymptotic nonbasis of order 2 for every finite set $S \subseteq \mathbf{N} \backslash A$, then $A$ contains a maximal asymptotic nonbasis of order 2.

Is every asymptotic basis of order $h$ a subset of a maximal asymptotic nonbasis of order $h$ ? No. Hennefeld [19] proved that for every $h \geq 2$ there exists an asymptotic nonbasis $A$ of order $h$ such that if $S \subseteq \mathbf{N} \backslash A$, then $A \cup S$ is an asymptotic nonbasis $A$ of order $h$ if and only if the set $\mathbf{N} \backslash(A \cup S)$ is infinite.

Investigating extremal properties of additive bases is like exploring for new plant species in the Amazon rainforest. Much has been collected, but much more is unimagined. The following results about oscillations of bases and nonbases appear in [9], [10].

There exists a minimal asymptotic basis of order 2 such that $A \backslash\{x\}$ is a maximal asymptotic nonbasis of order 2 for every $x \in A$.

There exists a maximal asymptotic nonbasis of order 2 such that $A \cup\{y\}$ is a minimal asymptotic basis of order 2 for every $y \in \mathbf{N} \backslash A$.

There exists a partition of the nonnegative integers into disjoint sets $A$ and $B$ such that $A$ is a minimal asymptotic basis of order 2 and $B$ is a maximal asymptotic nonbasis of order 2 .

There exists a partition of the nonnegative integers into disjoint sets $A$ and $B$ that oscillate in phase from minimal asymptotic basis of order 2 to maximal asymptotic nonbasis of order 2 as random elements are moved from the basis to the nonbasis infinitely often.

It is an open problem to extend these results to asymptotic bases of order $h \geq 3$. For a survey of extremal problems in additive number theory, see Nathanson [30].

\section{References}

[1] E. ARTIN and P. SCHERK, On the sum of two sets of integers, Annals Math. 44 (1943), 138-142.

[2] J. W. S. CASSELS, Über Basen der natürlichen Zahlenreihe, Abhandlungen Math. Seminar Univ. Hamburg 21 (1957), 247-257.

[3] J.-M. Deshouillers and G. Grekos, Propriétés extréales de bases additives, Bull. Soc. Math. France 107 (1979), 319-335.

[4] F. J. Dyson, A theorem on the densities of sets of integers, J. London Math. Soc. 20 (1945), 8-14.

[5] P. ERDŐS and P. TURÁN, On a problem of Sidon in additive number theory, and on some related problems, J. London Math. Soc. 16 (1941), 212-215.

[6] P. ERDÖS, On the arithmetical density of the sum of two sequences, one of which forms a basis for the integers, Acta Arith. 1 (1936), 197-200.

[7] _ On the multiplicative representation of integers, Israel J. Math. 2 (1964), 251-261.

[8] P. ERDős and M. B. NATHANSON, Maximal asymptotic nonbases, Proc. Amer. Math. Soc. 48 (1975), 57-60.

[9] __ Oscillations of bases for the natural numbers, Proc. Amer. Math. Soc. 53 (1975), 253-358.

[10] _ Partitions of the natural numbers into infinitely oscillating bases and nonbases, Commentarii Math. Helvet. 52 (1976), 171-182.

[11] _ Nonbases of density zero not contained in maximal nonbases, J. London Math. Soc. (2) 15 (1977), no. 3, 403-405.

[12] $\_$, Sets of natural numbers with no minimal asymptotic bases, Proc. Amer. Math. Soc. 70 (1978), 100-102.

[13] _ Systems of Distinct Representatives and Minimal Bases in Additive Number Theory, Number Theory, Carbondale, 1979 (Heidelberg) (M. B. Nathanson, ed.), Lecture Notes in Mathematics, vol. 751, Springer-Verlag, 1979, pp. 89-107.

[14] _ Minimal asymptotic bases with prescribed densities, Illinois J. Math. 32 (1988), 562-574.

[15] H. Halberstam and K. F. Roth, Sequences, Vol. 1, Oxford University Press, Oxford, 1966; Reprinted by Springer-Verlag, Heidelberg, 1983.

[16] E. HÄRTTER, Eine Bemerkung über periodische Minimalbasen für die Menge der nichtnegativen ganzen Zahlen, J. Reine Angew. Math. 214/215 (1964), 395-398.

[17] P. Hegedüs, G. PIroskA, and I. Z. RuzsA, On the Schnirelmann density of sumsets, Publ. Math. Debrecen 53 (1998), no. 3-4, 333-345.

[18] H. A. HELFGOTT, The ternary Goldbach conjecture is true, arXiv:1312.7748 [math.NT], 2013. 
[19] J. HenNefeld, Asymptotic nonbases which are not subsets of maximal asymptotic nonbases, Proc. Amer. Math. Soc. 62 (1977), 23-24.

[20] A. YA. Khinchin, Über ein metrisches Problem der additiven Zahlentheorie, Mat. Sbornik N.S. 10 (1933), 180-189.

[21] M. KNESER, Abschätzungen der asymptotischen Dichte von Summenmengen, Math. Z. 58 (1953), 459-484.

[22] E. LANDAU, Über einige neuere Fortschritte der additiven Zahlentheorie, Cambridge University Press, Cambridge, 1937.

[23] H. B. MANN, A proof of the fundamental theorem on the density of sums of sets of positive integers, Annals of Math. 43 (1942), 523-527.

[24] J. C. M. NASH and M. B. NATHANSON, Cofinite subsets of asymptotic bases for the positive integers, J. Number Theory 20 (1985), no. 3, 363-372.

[25] M. B. NATHANSON, Minimal bases and maximal nonbases in additive number theory, J. Number Theory 6 (1974), 324-333.

[26] _ Multiplicative representations of integers, Israel J. Math. 57 (1987), no. 2, 129-136.

[27] _ Best possible results on the density of sumsets, Analytic Number Theory (Allerton Park, IL, 1989), Progr. Math., vol. 85, Birkhäuser Boston, Boston, MA, 1990, pp. 395-403.

[28] _ Additive Number Theory: The Classical Bases, Graduate Texts in Mathematics, vol. 164, SpringerVerlag, New York, 1996.

[29] __ Cassels bases, Additive Number Theory, Springer, New York, 2010, pp. 259-285.

[30] _ Additive Number Theory: Extremal Problems and the Combinatorics of Sumsets, Graduate Texts in Mathematics, Springer, New York, to appear.

[31] M. B. NATHANSON and ANDRÁS SÁRKÖZY, On the maximum density of minimal asymptotic bases, Proc. Amer. Math. Soc. 105 (1989), 31-33.

[32] J. NEŠETR̆IL and V. RöDL, Two proofs in combinatorial number theory, Proc. Amer. Math. Soc. 93 (1985), 185188.

[33] H. PlüNNECKE, Über ein metrisches Problem der additiven Zahlentheorie, J. Reine Angew. Math. 197 (1957), 97-103.

[34] _ , Über die Dichte der Summe zweier Mengen, deren eine die dichte null hat, J. Reine Angew. Math. 205 (1960), 1-20.

[35] _ Eigenschaften und Abschätzungen von Wirkungsfunktionen, vol. 22, Berichte der Gesellschaft für Mathematik und Datenverarbeitung, Bonn, 1969.

[36] D. RAIKOV, Über die Basen der natürlichen Zahlentreihe, Mat. Sbornik N.S. 244 (1937), 595-597.

[37] I. Z. RuzsA, Essential components, Proc. London Math. Soc. 54 (1987), 38-56.

[38] L. G. SHNIREL'MAN, On the additive properties of integers, Izv. Donskovo Politekh. Inst. Novocherkasske 14 (1930), 3-27.

[39] _ Über additive Eigenschaften von Zahlen, Math. Annalen 107 (1933), 649-690.

[40] A. STÖHR, Eine Basis $h$-Ordnung für die Menge aller natürlichen Zahlen, Math. Zeit. 42 (1937), 739-743.

[41] _ Gelöste und ungelöste Fragen über Basen der natürlichen Zahlenreihe. I, II, J. Reine Angew. Math. 194 (1955), 40-65, 111-140.
American Mathematical Society

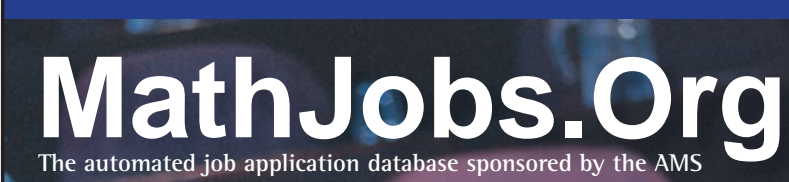

The automated joh application datahase snonsored hy the AMS

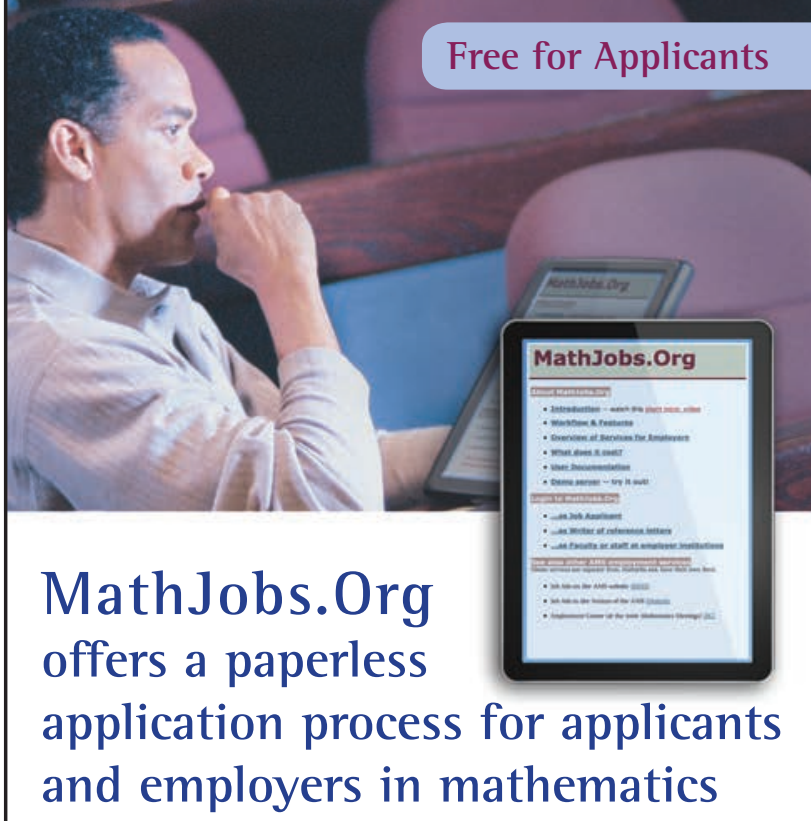

\section{Registered Applicants Can:}

- Create their own portfolio of application documents

- Make applications online to participating employers

- Choose to make a cover sheet viewable by all registered employers

\section{Registered Employers Can:}

- Post up to seven job ads

- Set all criteria for required documents, and add specific questions

- Receive and upload reference letters

- Manage applicant information and correspondence quickly and easily

- Set limited access permissions for faculty and EOE administrators

- Search for and sort additional applicants in the database

- Choose an advertising-only account, or a discounted single ad account

\section{Visit mathjobs.org for pricing information}

Contact: Membership and Programs Department

American Mathematical Society

201 Charles Street

Providence, Rl 02904-2294 USA

800.321.4267, ext. 4105

Email: mathjobs@ams.org

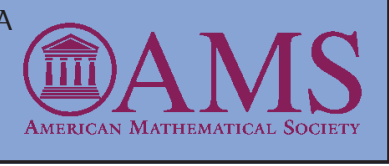

